\title{
Routine screening for intimate partner violence: A guide for trainers
}

Odongo Odiyo

Chi-Chi Undie

Population Council

Margaret Mak'anyengo

Follow this and additional works at: https://knowledgecommons.popcouncil.org/departments_sbsr-rh

Part of the Demography, Population, and Ecology Commons, Domestic and Intimate Partner Violence Commons, Family, Life Course, and Society Commons, Health Policy Commons, International Public Health Commons, Maternal and Child Health Commons, and the Women's Health Commons How does access to this work benefit you? Let us know!

\section{Recommended Citation}

Odiyo, Odongo, Chi-Chi Undie, and Margaret Mak'anyengo. 2016. "Routine screening for intimate partner violence: A guide for trainers." ECSA Health Community, Population Council, and Kenyatta Hospital. 


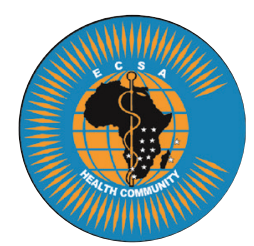

East, Central and Southern

Africa Health Community

Fostering Regional Cooperation for Better Healt

ECSA Health Community Secretariat

157 Olorien, Njiro Road

P.O Box 1009, Arusha, Tanzania

The East, Central and Southern African Health Community (ECSA-HC) is a regional inter-governmental health organization that fosters and promotes regional cooperation in health among member states.

Member states of the ECSA Health Community include Kenya, Lesotho, Malawi, Mauritius, Seychelles, Swaziland, United Republic of Tanzania, Uganda, Zambia and Zimbabwe.

ECSA-HC was established in 1974 to foster and strengthen regional cooperation and capacity to address the health needs of the member states. Through partnerships with diverse institutions, ECSA's activities also spread to other countries in Africa to address common health challenges facing the region. The ECSA Health Community works with countries and partners to raise the standard of health for the people of the ECSA region by promoting efficiency and effectiveness of health services through cooperation, collaboration, research, capacity building, policy development and advocacy.

Suggested citation: Odongo Odiyo, Chi-Chi Undie, and Margaret Mak'anyengo. 2016. "Routine screening for intimate partner violence: A guide for trainers." ECSA Health Community, Population Council, and Kenyatta Hospital. 


\section{Contents}

Foreword $\quad$ iv

Preface $\quad v$

Acknowledgments vi vi

$\begin{array}{ll}\text { PART ONE } & 1\end{array}$

Session 1: Who's in the room? (Welcome and introduction of participants) 2

Session 2: Reality Check: Unpacking IPV

Session 3: Testimonial: A Survivor's Story 7

$\begin{array}{ll}\text { PART TWO } & 9\end{array}$

Session 1: IPV: What is it? And how can we help? $\quad 10$

Session 2: Nuts and bolts: IPV screening and referral $\quad 12$

Session 3: This is what it feels like: Role play 14

Session 4: Discovery: Getting to know your GBV clinic 16

Session 5: Planning to act: Developing an action plan for routine IPV screening 18

Session 6: What we've learned and how it went: Closing 20

$\begin{array}{ll}\text { APPENDIXES } & 23\end{array}$

Appendix A: Traffic light exercise $\quad 24$

Appendix B: Caged bird exercise $\quad 35$

Appendix C: PowerPoint Presentation Slides (with notes pages) 37

Appendix D: Handouts $\quad 47$ 


\section{Foreword}

Global and regional estimates of violence against women released in 2013 by the World Health Organization (WHO), the London School of Hygiene and Tropical Medicine, and the South African Medical Research Council confirm that intimate partner violence (IPV) represents the most common form of violence experienced by women worldwide, and that the prevalence of physical and/or sexual IPV in the African region is particularly high among ever-partnered women (at 37\%).

To combat this common form of violence, researchers have increased experimentation with clinic-based IPV interventions, coupled with high-impact meetings in the region to explore how IPV screening interventions are being conducted and what lessons can be learned for strengthening these endeavors. There is growing recognition by health providers of the associations between IPV and the health conditions with which their clients present. Furthermore, health providers and their institutions are acknowledging that incorporating IPV screening protocols into their practice could potentially enhance the quality of care for their clients, while reducing provider workloads by helping to more rapidly pinpoint the root cause of many clients' health problems. In addition, recent research demonstrates the acceptability and feasibility of IPV screening in the region, and showcases the ability of providers play a key role in screening.
Spurred by these contextual realities, in December 2012 Health Ministers from the East, Central and Southern Africa (ECSA) region passed a resolution that calls for the integration of gender-based violence screening into sexual and reproductive health and HIV and AIDS services in ECSA countries, coupled with support for Member States to effect such integration (ECSA/HMC56/R2, Number 5 [ECSA Secretariat] and Number 7 [ECSA Member States]). Operationalizing this resolution will entail a combination of efforts, including developing guidance for service providers on modalities for carrying out screening. This guide represents a key step in this direction to promote momentum in this area.

Given the ECSA Heath Ministers' resolution on screening for gender-based violence, the ECSA Health Community envisions that this training guide will facilitate the integration of screening protocols in health care settings, thus contributing to the improvement of health sector efforts in the area of gender-based violence response and prevention. Member states are urged to use this guide as an important means of addressing gender-based violence in the region.

PROF. YOSWA DAMBISYA

Director General ECSA Health Community 


\section{Preface}

Routine Screening for Intimate Partner Violence: A Guide for Trainers is the first regional document devoted to building the capacity of providers to respond to intimate partner violence (IPV). The training guide is a follow-on initiative to the $6^{\text {th }}$ Best Practices Forum in Health of the East, Central and Southern Africa (ECSA) Health Community, held in August 2012. This Forum brought to the fore research findings from the region centering on the acceptability and feasibility of IPV screening, and culminated in the passage of a resolution (in December 2012) by Health

Ministers that prescribes the integration of gender-based violence screening into health care settings in the ECSA region. This training guide responds to this resolution by providing Member States with a starting point for implementing the proposed integration of gender-based violence screening.

The document has also been developed in recognition of the growing demand for practical materials to guide the introduction of IPV screening protocols into various programs. It is designed to be simple and easily adaptable to various provider types and service provision contexts and goals. First piloted in 2012 within several clinics at the Kenyatta National Hospital, Kenya, the training guide is also designed to be brief (a day long), given the impracticalities of withdrawing providers from their work settings for extended time periods.

We anticipate that this toolkit will serve as a valuable resource for a range of service providers who work to prevent and respond to gender-based violence, and we are pleased to invite Member States and other partners to adopt the guide, or to adapt it according to their needs to build capacity and enhance the quality of care in the ECSA region. 


\section{Acknowledgments}

This training guide was made possible by a grant from the Regional Team for Sexual and Reproductive Health and Rights, Embassy of Sweden, Lusaka, Zambia.

The interactive exercises found in Session Two of this training guide were adapted from: Ellsberg M. and Heise L. Researching Violence Against Women: A Practical Guide for Researchers and Activists. PATH, World Health Organization, 2005. Hand-out \#3 ('General tips for IPV screening') as well as some of the material used in the PowerPoint presentation that supports this training guide, was adapted from the 1996 publication entitled Improving the Health Care System's Response to Domestic Violence: A Resource Manual for Health Care Providers, produced by the Family Violence Prevention Fund in collaboration with the Pennsylvania Coalition Against Domestic Violence, and written by Carole Warshaw, M.D. and Anne L. Ganley, Ph.D., with contributions by Patricia R.

Salber, M.D. We gratefully acknowledge the support of the Population Council's office of publishing and creative services in designing and editing this document. 


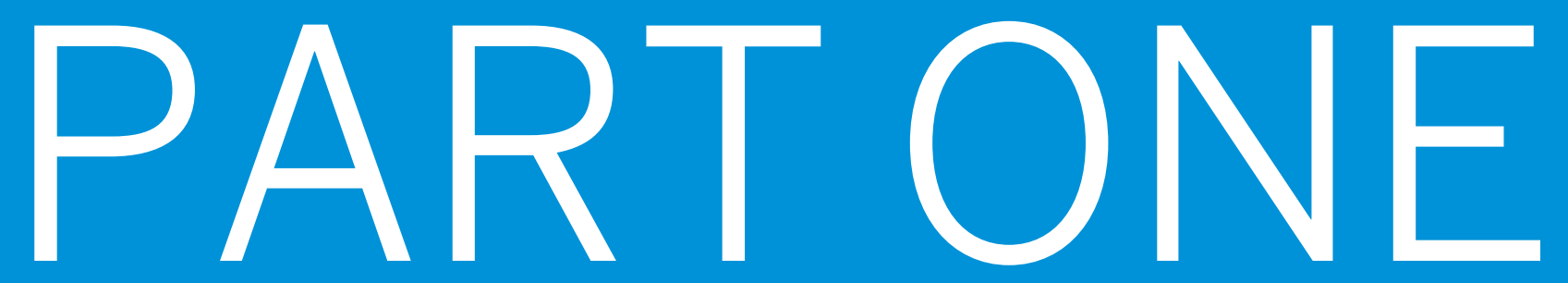




\section{SESSION}

1

\section{Who's in the room?}

Welcome and introduction of participants

OBJECTIVES
- To give participants a chance to get to know
one another
- To give the facilitator a sense of who the
participants are (Are they all from the
same department/clinic? Are there a mix of
participants from different departments/
clinics?)
- To foster an open, relaxed, non-threatening
environment for the training session through
having participants share a personal
memory
To share the overall objectives of the training
session

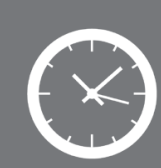

DURATION 30 MINUTES

(about 1

minute per participant, with an additional 5 minutes built in)

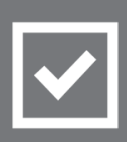

ADVANCE PREPARATION

- Ensure that the total number of coins available for this exercise comes to about twice the total number of expected participants. If finding coins from different countries proves challenging, simply ensure that the available coins are in different denominations and were not all minted in the same year.

- Place one coin in each participant's package of materials beforehand. Place each coin in the same area in each package for easy retrieval.

- Set aside a coin for your own introduction.

- Ensure that there is representation (among participants) from the GBV clinic to which IPVpositive clients will be referred once screening protocols are in place. 


\section{PROCESS}

\section{STEP 1}

1. Thank participants for making the time to attend the session and explain that a fun/interesting activity will be used to facilitate introductions.

2. Point out that every participant has a coin in his/her package, and let participants know exactly where this may be found.

3. Start by introducing yourself - your name, affiliation, and position. Then, look at the back of your own coin for the year in which the coin was minted. Announce the year to the participants and share one memory you have of that particular year.

4. Ask participants to introduce themselves one after the other in the same fashion: name, affiliation and position (institution and department - if participants were invited from different institutions/departments), year on the back of coin, and one memory from that year.

\section{STEP 2}

1. Share the overall objective of the training: to sensitize providers on the issue of IPV among their clients, its health implications, and the role that providers can play in addressing it.

2. Emphasize to participants that this is a 'training and planning' session - a two-way, interactive process through which the facilitator and the participants learn from one another. While the facilitator provides training on how providers can respond to IPV, providers teach the facilitator about their individual work contexts and how responses can be shaped in these settings to ensure intervention success.

\section{FACILITATOR NOTES}

The training session is designed for no more than 20-25 participants so that the exercises are manageable and to ensure optimal engagement of participants during small-group and plenary sessions. The facilitator is advised to be prepared for surprises. Some common surprises may have to do with the fact that some unanticipated participants may attend the training (e.g., staff from departments that were not invited for the training) - or with the fact that not all memories shared during the introductions will be 'fun.' Some coin dates may remind participants of sad events (e.g., the death of a loved one). Be prepared with something brief and appropriate to say. Make mental or written notes of participants' affiliations and positions, as this will help in rectifying any anomalies with Heads of Department later, and will help in understanding or anticipating group dynamics that may provide opportunities or pose challenges for impending sessions, and for intervention planning. 


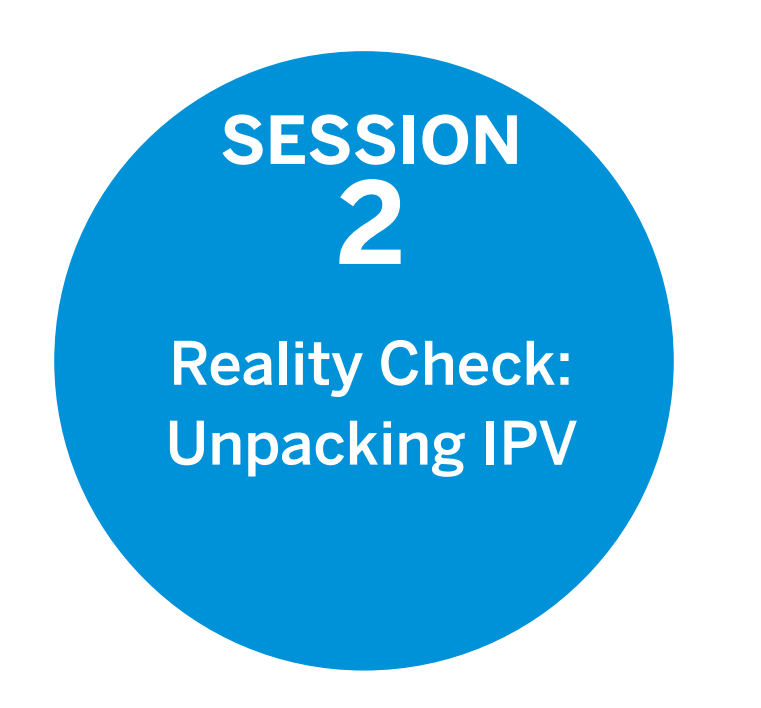

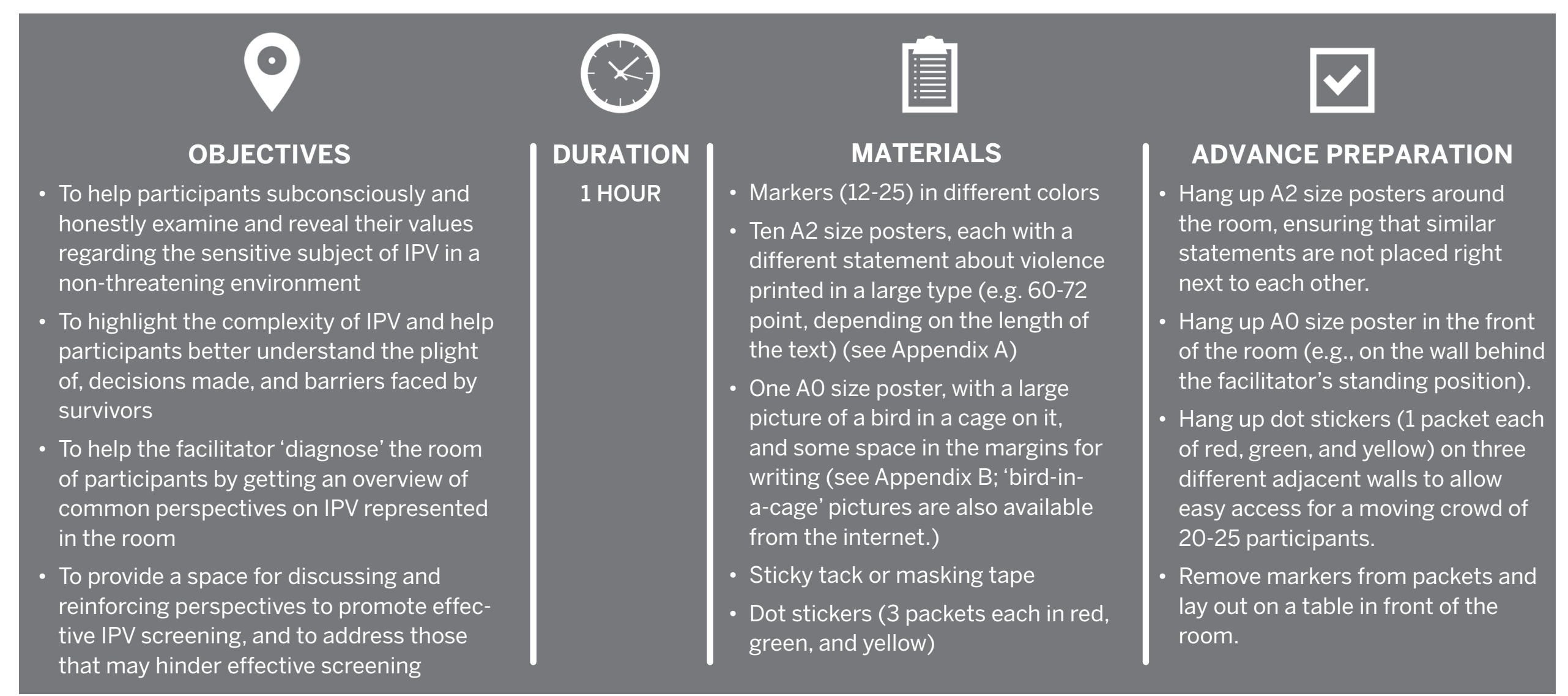




\section{STEP 1}

1. Point out to participants that around the room are 10 different statements for which there are not necessarily any 'right' or 'wrong' answers, and which will be used as part of a warm-up exercise referred to as the 'Traffic Light' exercise.

2. Instruct participants to walk round the room, making sure to visit all 10 posters. At each poster, they are to read the statement and decide whether they agree, disagree, or are undecided about it. They should put one sticker on each poster according to their honest opinion: a green sticker for 'agree, a red one for 'disagree,' and a yellow one for 'undecided.

3. Encourage participants to move quickly from one poster to the next without overthinking their responses. Emphasize that if they are not completely sure of what they think about a particular statement, they should simply put up a yellow dot sticker on the poster concerned.

\section{STEP 2}

1. Draw participants' attention to the $\mathrm{AO}$ size poster of the caged bird at the front of the room. The poster is captioned with the title, 'Why Doesn't She Just Leave?'

2. Explain that the caged bird symbolizes a woman in an abusive relationship. Despite the abuse, she feels trapped within the relationship, much like the bird in the cage. Ask participants to take a moment to think about why this woman does not just leave the relationship. Instruct participants to walk up to the poster, pick up a marker, and write the first answer that comes to mind on the poster. Each participant should write down only one answer on the poster.
3. Ask participants to settle down for a discussion of the two activities.

\section{STEP 3}

1. Have participants take a minute to look around the room at the $10 \mathrm{~A} 2$ size posters and provide an opportunity for them to comment on any striking patterns they see in regard to the colored dots. Use each comment to convey and reinforce messages about the complexity, possible drivers, misconceptions, and consequences of IPV.

2. Participants should be given an opportunity to comment on all 10 posters. Afterward, point out that this exercise is useful in bringing our own biases about IPV to the fore, and that this process is important for providers, as notions about IPV can either hinder or promote effective IPV screening.

3. Draw participants' attention to the AO size poster. Read some of the comments written on it. Have participants volunteer to elaborate further on the comments and what they mean. Where possible, use participants' comments to reinforce messages about the complexity of IPV, and about the fact that a woman experiencing this form of violence often has very legitimate reasons for remaining in the abusive relationship

4. Wrap up the discussion with the message that the bird in the cage symbolizes many of the women who will be identified through the IPV screening intervention that will be discussed in later sessions of the training, and that, as providers, it is important to understand where these women are coming from, and to care for them with a non-judgmental attitude. 


\section{FACILITATOR NOTES}

These lively, interactive exercises are best done in a room with enough space available for participants to walk around, view each poster, and access the dot stickers or markers as conveniently and quickly as possible. The facilitator will need to be prepared to highlight one or two observable patterns to get the discussion going before encouraging participants to follow suit. For example, on the A2 size poster with the statement 'Men are violent by nature,' there may be a mix of colored dots (e.g., red, green, and yellow) by the end of the exercise. This dot pattern indicates the mixed opinions of the participants about the statement: some believe that men are violent by nature, some do not, and others are not sure. In such a case, the facilitator would point out to participants that if the beliefs in the room on this issue are not resolved, they could have implications for the IPV screening process. A provider who believes that men are violent by nature would not be motivated to screen clients for intimate partner violence - after all, violence is 'natural' in such a provider's mind, and it would therefore be futile to try to respond to it. These are the kinds of discussions that the two interactive exercises are meant to provoke, and these are the kinds of issues that the facilitator must look out for and highlight in order to resolve them through group discussion. 


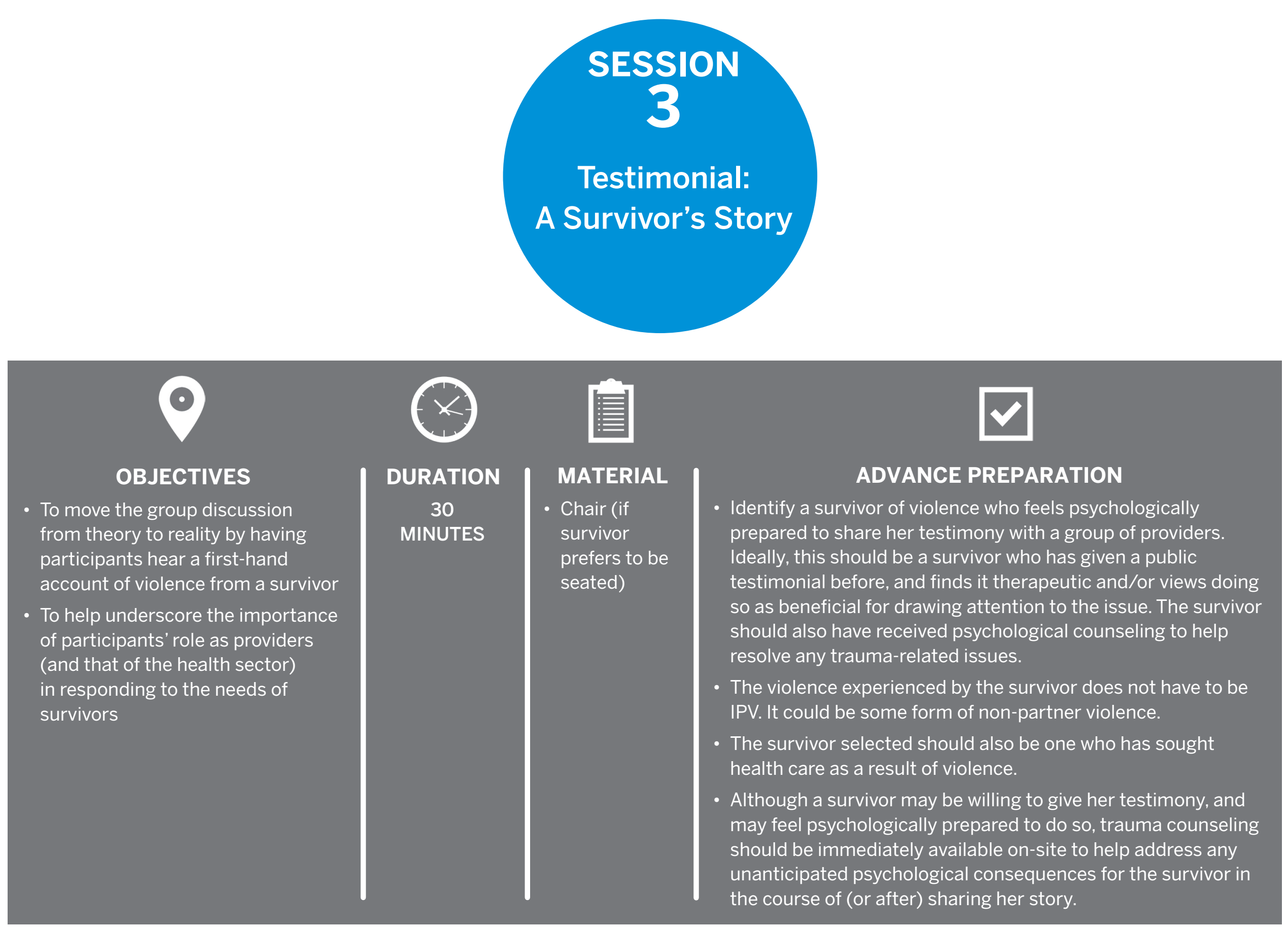




\section{STEP 1}

1. Inform participants that they are about to have a rare opportunity to hear and learn from a special guest. Explain that the guest will bring to life the issues that the training session has dwelt on all morning.

2. Caution participants about the need to be respectful of and sensitive toward the guest and her testimony.

3. Ask participants to note down the thoughts that come to mind about the role of providers in responding to the needs of survivors. Have them take note of areas where the health system and its providers worked well, and worked less well.

4. Invite the survivor to introduce herself (she may choose to remain anonymous), and to share her story.

5. Ensure the survivor is given a warm welcome both by the facilitator and the audience (e.g., applause).

\section{STEP 2}

1. Give the speaker 10-15 minutes to share her testimony.

2. Remain alert during the testimony in order to take note of specific parts of the survivor's story that have an effect on participants.

3. Encourage applause from the participants at the end of the testimony.

4. Ask participants if they have any comments or questions for the survivor or larger group. Emphasize that remarks should not be accusatory or offensive toward the survivor, but should rather focus on helping the group reflect on the health system, their roles as providers, what could have been done differently, or on good practices to be commended.

5. Ask participants what they have learned from the testimony, or what they are taking home from the survivor's story.

6. If necessary, the facilitator can bring up parts of the story s/he noted down as affecting the participants (see number 2 above) in order to stimulate discussion.

7. Wrap up the session with a statement of appreciation for the survivor's time, and with further applause.

\section{FACILITATOR NOTES}

Well in advance, the survivor should be advised on who her audience will be, the role of her testimony in the training session, how the training room will be set up, and all that is expected of her. The survivor should be given the option of participating (anonymously) in Session Two in order to gain a feel for the kind of participants she will be addressing, and to incorporate issues that emerged from the interactive exercises into her testimony. from the interactive exercises during her testimony. The survivor should be prepared to devote $10-15$ minutes to sharing her testimony, and another 1015 minutes to addressing comments/ questions from participants. 


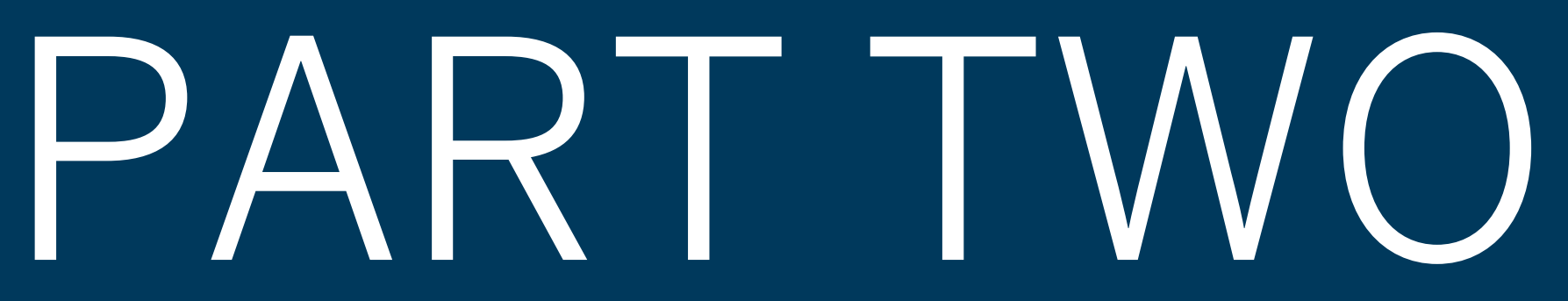




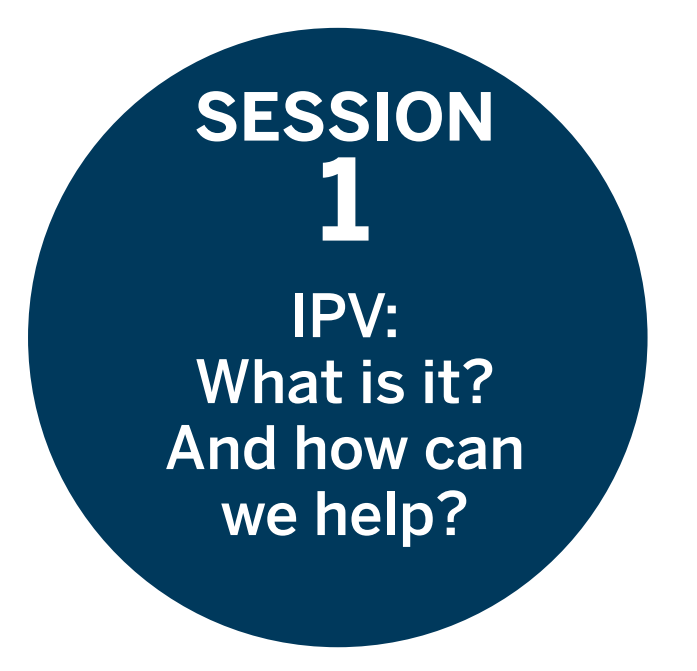

OBJECTIVES
$\begin{aligned} & \text { To explore formal } \\ & \text { definitions of IPV and } \\ & \text { the rationale for IPV } \\ & \text { screening }\end{aligned}$
$\begin{aligned} & \text { To introduce the } \\ & \text { planned IPV screening } \\ & \text { intervention, and the } \\ & \text { participants' role within } \\ & \text { it }\end{aligned}$




\section{PROCESS}

\section{STEP 1}

1. Beginning from Slide 1, go over each slide with participants.

2. For each slide, refer to the presentation notes (see Appendix C), whenever necessary.

3. After presenting each slide (or during this process), invite participants to share any questions or comments they may have before moving on to the next.

\section{FACILITATOR NOTES}

A PowerPoint presentation is available in the Appendix to guide the facilitation of Part Two of the training session. Each presentation slide comes with comprehensive notes for the facilitator (see the Notes Page on each presentation slide in the Appendix). The slides and notes are meant to be adaptable, and should be edited or adapted ac- cording to the planned screening intervention (e.g., screening for sexual and gender-based violence in its most comprehensive form, or screening for physical intimate partner violence only, or screening for non-partner sexual violence only). 


\section{SESSION}

2

Nuts and bolts:

IPV screening

and referral

OBJECTIVES
$\begin{aligned} & \text { To explore and collaboratively } \\ & \text { make plans with participants } \\ & \text { for the practical side of IPV } \\ & \text { screening, including how to: } \\ & \text { ask screening questions and } \\ & \text { refer such clients for further } \\ & \text { care }\end{aligned}$
$\begin{aligned} & \text { - To shed light on the } \\ & \text { psychology of some survivors } \\ & \text { that may prevent immediate } \\ & \text { disclosure, and on the role of } \\ & \text { routine screening in making } \\ & \text { survivors comfortable enough } \\ & \text { to eventually disclose IPV }\end{aligned}$
$\begin{aligned} & \text { - Hand-outs: } \\ & \text { IPV Screening- }\end{aligned}$




\section{PROCESS}

\section{STEP 1}

1. Beginning from Slide 7, go over each slide with participants.

2. For each slide, refer to the presentation notes (see Appendix C), whenever necessary.

3. After presenting each slide (or during this process), invite participants to share any questions or comments they may have before moving on to the next.

\section{FACILITATOR NOTES}

As indicated on the cover slide of the

PowerPoint presentation, this forum is a 'training and planning' session, which implies a collaborative process between the facilitator and the participants, who have expert knowledge about their own clinics/departments, and who therefore have an understanding of the best way to set up a routine IPV screening protocols in their own work contexts. In Session Two, the facilitator is encouraged to foster a participatory atmosphere, guiding participants to consider how screening and referral would work optimally in their settings.

This session involves the use of several hand-outs, which should be reviewed as the relevant presentation slides come up. The PowerPoint presentation notes pages indicate when to draw participants' attention to each hand-out. 


\section{SESSION}

\section{3}

\section{This is what}

it feels like:

Role play

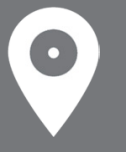

OBJECTIVES

- To provide participants with the opportunity to 'walk in the shoes' of key players in the IPV screening process: the client and the provider

- To provide space for participants to familiarize themselves with the screening and referral process

- To improve screening procedures by having participants reflect on the process as actors and observers

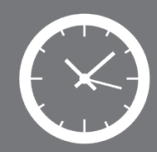

DURATION 30 MINUTES

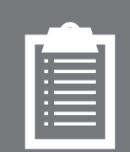

MATERIALS

- Laptop

- Projector

- Pens

- Paper/note pads

- Hand-out:

IPV screening tool/ questions

\section{网}

\section{ADVANCE PREPARATION}

- Place a pen and note-pad in participants' training folders to save time

- File IPV screening tool hand-out in participants' training folders in the order in which it is to be used during this session

- Become familiar with the presentation notes and hand-out to ensure a smooth, comfortable delivery 


\section{PROCESS}

\section{STEP 1}

1. Put up Slide 13.

2. Explain that all participants will be taking part in a role play exercise, and will need to get into groups of 3. Put up Slide 14 (1 $1^{\text {st }}$ bullet).

3. Once groups are formed, explain that each person will get a chance to play three roles: provider, client, and observer. The provider will conduct the actual screening by posing the IPV screening questions to the client; the client will respond to the screening questions; and, the observer will take note of events or processes that the provider and client may not be able to, given their focus on their individual roles. These observations can be written down as they will be shared with the wider group later.

4. Each participant in the groups of 3 should take turns playing each role - i.e., the role of provider, client, and observer.

\section{STEP 2}

1. Once participants have completed the above process, move the session back into plenary, and ask for any comments or observations anyone may have about the exercise.

2. After preliminary comments and observations have been addressed, put up Slide 14 (2nd bullet) to generate specific discussion on how participants felt playing each role.

3. Discuss and collaboratively address any issues related to:

a. discomfort in asking the questions, and the role of the preamble to the screening questions in reducing potential discomfort for providers and clients alike

b. discomfort in being asked the questions, and what this means for provider attitudes and presentation of the questions

c. any other observations that observers and other roleplayers may have, such as areas of improvement for providers asking the questions; possible issues that could arise as a result of how clients responded to the questions, and how these can be addressed; any issues concerning the translation of the screening questions into the local language (if applicable), etc.

\section{FACILITATOR NOTES}

It should take about 10-15 minutes for the small-group exercise to take place. The facilitator may find it useful to spend this time by walking around the room to unobtrusively observe and listen to various groups in order to derive some talking points for the plenary session. These can be used to stimulate discussion during the plenary session, if necessary. 


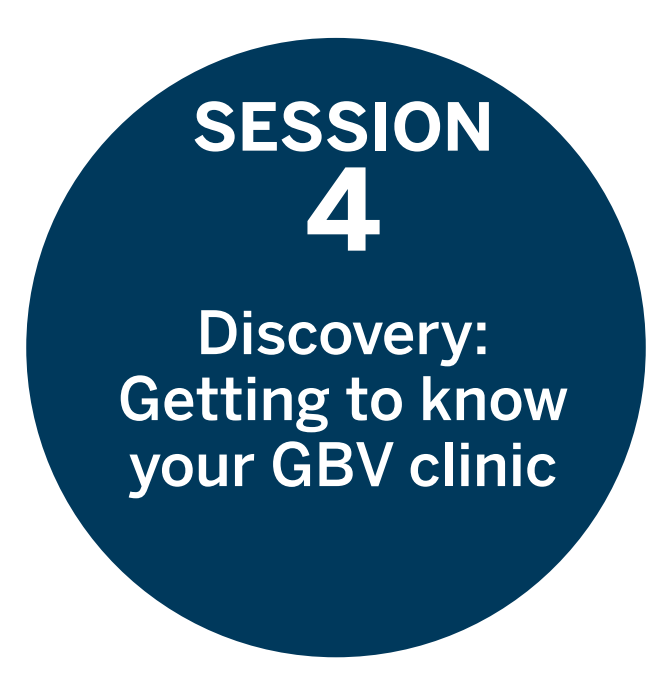

OBJECTIVES
To provide participants with
the opportunity to visit a GBV
$\begin{aligned} & \text { clinic (on-site, if available; off- } \\ & \text { site, if not) }\end{aligned}$
$\begin{aligned} & \text { To create awareness among } \\ & \text { (could be } \\ & \text { shortened to } \\ & \text { services }\end{aligned}$
$\begin{aligned} & \text { To minutes if } \\ & \text { the GBV clinic } \\ & \text { is on-site) } \\ & \text { the needs of clients identified } \\ & \text { through screening can and will } \\ & \text { be addressed once referrals } \\ & \text { are made }\end{aligned}$




\section{PROCESS}

\section{STEP 1}

1. Explain the purpose of the visit to participants

2. Before embarking on the visit, alert participants to the need to be respectful of any survivors who may be present (e.g., in waiting areas) during the visit, by avoiding direct eye contact, as well as any communication

3. Emphasize the total amount of time allocated for this visit, in order for participants to be succinct with any comments or questions they may have for GBV clinic staff during the visit

\section{STEP 2}

1. After the visit, a few minutes may be used for further discussion of any barriers that participants feel survivors may face with referrals (i.e., getting to the GBV clinic) and how they can be addressed.

\section{FACILITATOR NOTES}

For various reasons, providers are often not aware of GBV services available, even when offered within their own work setting. Therefore, for many participants, this training session will be their first opportunity to visit and learn about a GBV clinic. The visit is important, as participants often wonder what happens to survivors after they are referred for further care. The visit can help reassure providers that services and competent staff are available, and that screening is an entry point to receiving these benefits. The specific GBV clinic to which survivors are to be referred should serve as the visit site for this session.
This session was designed on the assumption that an on-site GBV clinic would be available for survivors to be referred to. In fact, the original training sessions which led to the development of this manual took place in a seminar room within the Gender-Based Violence Recovery Centre at Kenyatta National Hospital. This location helped ensure that walking through this GBV clinic, and giving participants a chance to ask questions, took no more than 40 minutes. This exercise can easily be adapted for off-site GBV clinics, however, if survivors are to be referred to such sites after screening. In this case, the facilitator might consider moving the timing of this particular session to the very end of the training, as the visit (coupled with transportation time) would most likely take an hour or more.

Because of the assumption that the GBV clinic associated with the screening intervention will be on-site, the text box above indicates that no materials are needed. However, if the GBV clinic is not on-site, transportation may be required, and will thus need to be arranged in advance. 


\section{SESSION}

5

\section{Planning to act:}

Developing an

action plan for

routine IPV

screening

\section{$\odot$}

\section{OBJECTIVES}

- To provide space for participants to reflect on the following within their clinics/departments:

- existing resources within that could be used to support the IPV screening process

- potential barriers to the screening process

- overlooked personnel who would be an asset to the screening process, if trained (or in some other capacity beyond actual screening)

- To collaboratively find solutions (through plenary discussions) to barriers and other issues identified

- To underscore the fact that actual routine IPV screening will be commencing in their clinics/ departments, and the importance of preparing their individual work contexts for this intervention as a result

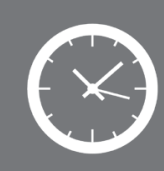

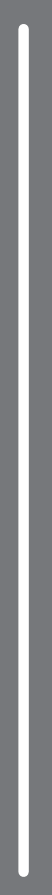

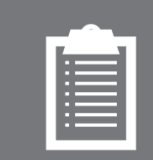

DURATION 45 MINUTES

\section{MATERIALS}

- Pens

- Hand-out:

Action plan

worksheet

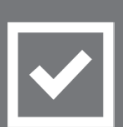

\section{ADVANCE PREPARATION}

- Place a pen in participants' training folders to save time

- File action plan worksheet handout in participants' training folders in the order in which it is to be used during this session

- Become familiar with the presentation notes and hand-out to ensure a smooth, comfortable delivery 


\section{PROCESS}

\section{STEP 1}

1. Have participants pull out the action plan worksheet

2. Walk participants through the worksheet, explaining the purpose and provide examples of the kind of responses expected in each section

3. Instruct participants to group themselves according to their clinics/departments, and to fill in the worksheets independently

4. Following this, participants are to discuss their sheets in the groups and come to consensus on any issues, such as possible 'allies' or 'opposition' within their clinics/ departments (see action plan worksheet)

\section{STEP 2}

5. Once participants have completed the above process, move the session back into plenary, and ask for a representative from each clinic/department to summarize their group discussions

6. Any unresolved issues noted during the presentation of each clinic/department should be collaboratively examined and addressed by the wider group to the extent possible

7. Ask participants to pass in their completed worksheets, as these will be a useful resource for any final planning that will need to be done for the screening intervention in each department

8. Staple the worksheets from each department together to maintain organization

\section{FACILITATOR NOTES}

This session was designed on the assumption that providers from different clinics will be trained together. Should this not be the case, the entire session can simply be held as a plenary session. It should take about 15 minutes for the worksheet completion portion of the exercise to take place. The facilitator may find it useful to spend this time listening in unobtrusively on participants' small group sessions or conversations. Important issues gleaned from this process can be used to stimulate discussion during the plenary session, if necessary. As participants report back from their groups (or from the plenary session), take detailed notes on any important observa- tions that arise. These observations, coupled with participants' completed action plan worksheets, can be referred to later to help in finalizing planning for the screening intervention. 


\section{SESSION}

6

\section{What we've learned}

and how it went:

Closing

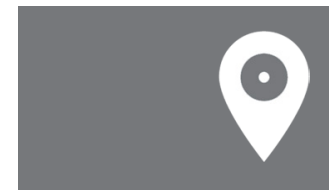

OBJECTIVES

- To gain an

understanding of what

participants found

most valuable about

the training session

- To collate participants opinions and

observations about

the training session

in order to inform and

help improve future

training sessions
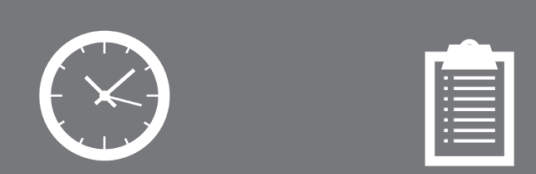

$\left|\begin{array}{|l|l|}\text { DURATION } & \text { MATERIALS } \\ 30 \text { MINUTES } & \begin{array}{l}\text { Pens } \\ \cdot \text { Hand-out: } \\ \text { Evaluation form }\end{array} \\ & \end{array}\right|$

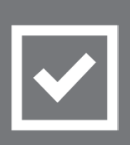

ADVANCE PREPARATION

- Place a pen in participants' training folders to save time

- File evaluation form hand-out in participants' training folders in the order in which it is to be used during this session

- Become familiar with the hand-out to ensure a smooth, comfortable delivery 


\section{PROCESS}

\section{STEP 1}

1. Express appreciation for participants' time, for what you have learned, and for all their contributions to defining how the IPV screening intervention will play out

2. Call on each participant one after the other to briefly highlight one thing that they will take away from the training-planning session, or to share any closing reflections they may have

\section{STEP 2}

1. Refer participants to the simple, anonymous evaluation form in their training folders and have them fill it out and turn it in

2. Explain to participants that they are free to leave once they have completed the evaluation form

\section{FACILITATOR NOTES}

Write participants' observations of the one thing they are taking away from the session, as well as any other reflections shared during this exercise. These observations can be used to inform future training sessions. Be sure to take note of, and share, what you have learned during the session.
Inform participants of when exactly the routine IPV screening intervention will commence in their clinics/departments, if possible. 

APPENDICES 


\section{Appendix A: Traffic light exercise}

Print out each of the statements on the next 10 pages on A2 size paper.

Examples:
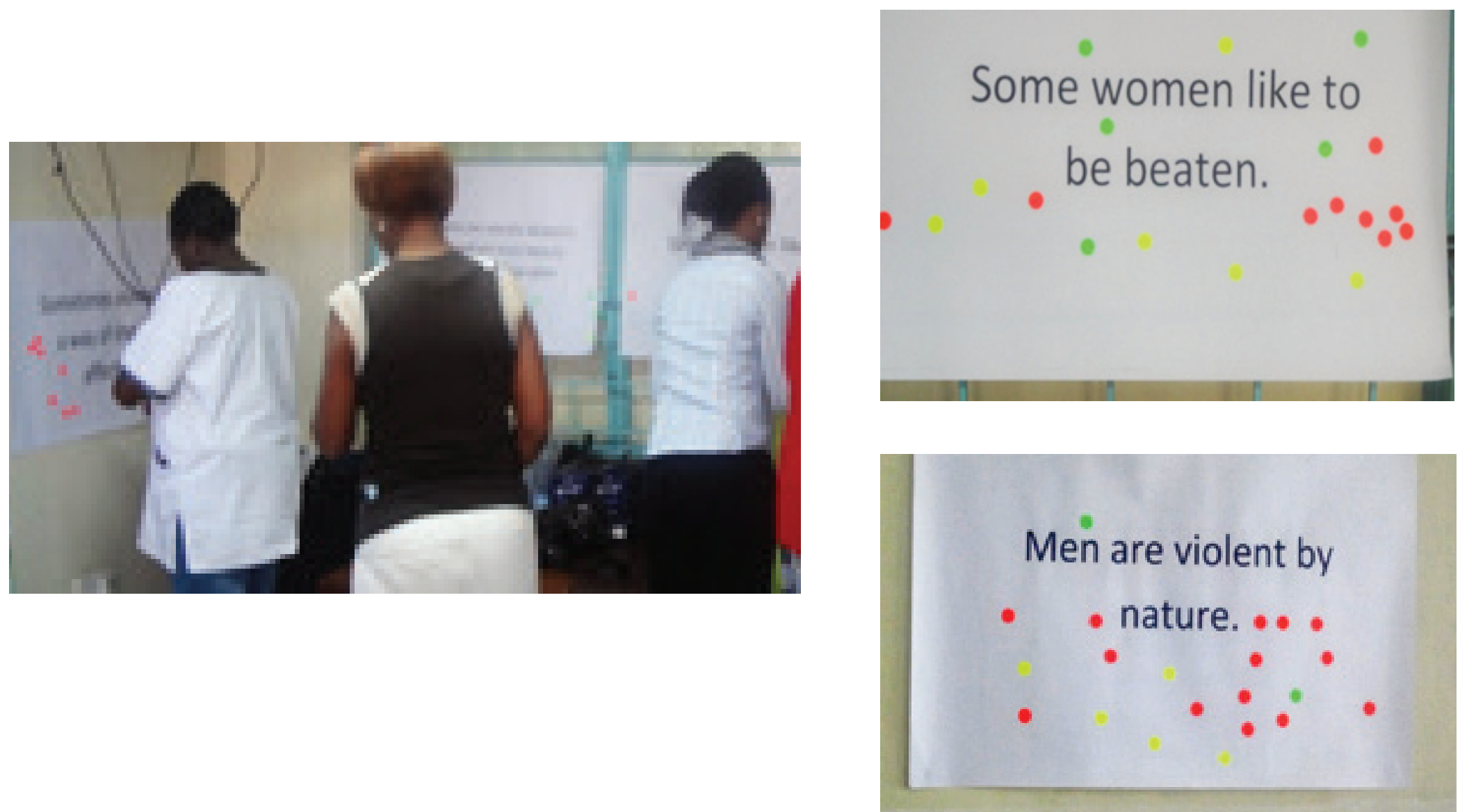
Men are violent by nature 
Sometimes violence is a way of showing affection 


\section{Boys who witness their}

father's violence toward their mother are more likely to be violent when they grow up 
A woman should put up with violence in order to

keep her family together 


\section{Some women like to be beaten}


Violence against women exists in every society in the world 
Nobody deserves to be raped 
Violence is never justified 
Nobody deserves to be beaten 
Girls who are sexually abused in childhood are

more likely to drink and use drugs when they're older 
Appendix B: Caged bird exercise

Print out the next page on $A O$ size paper. 


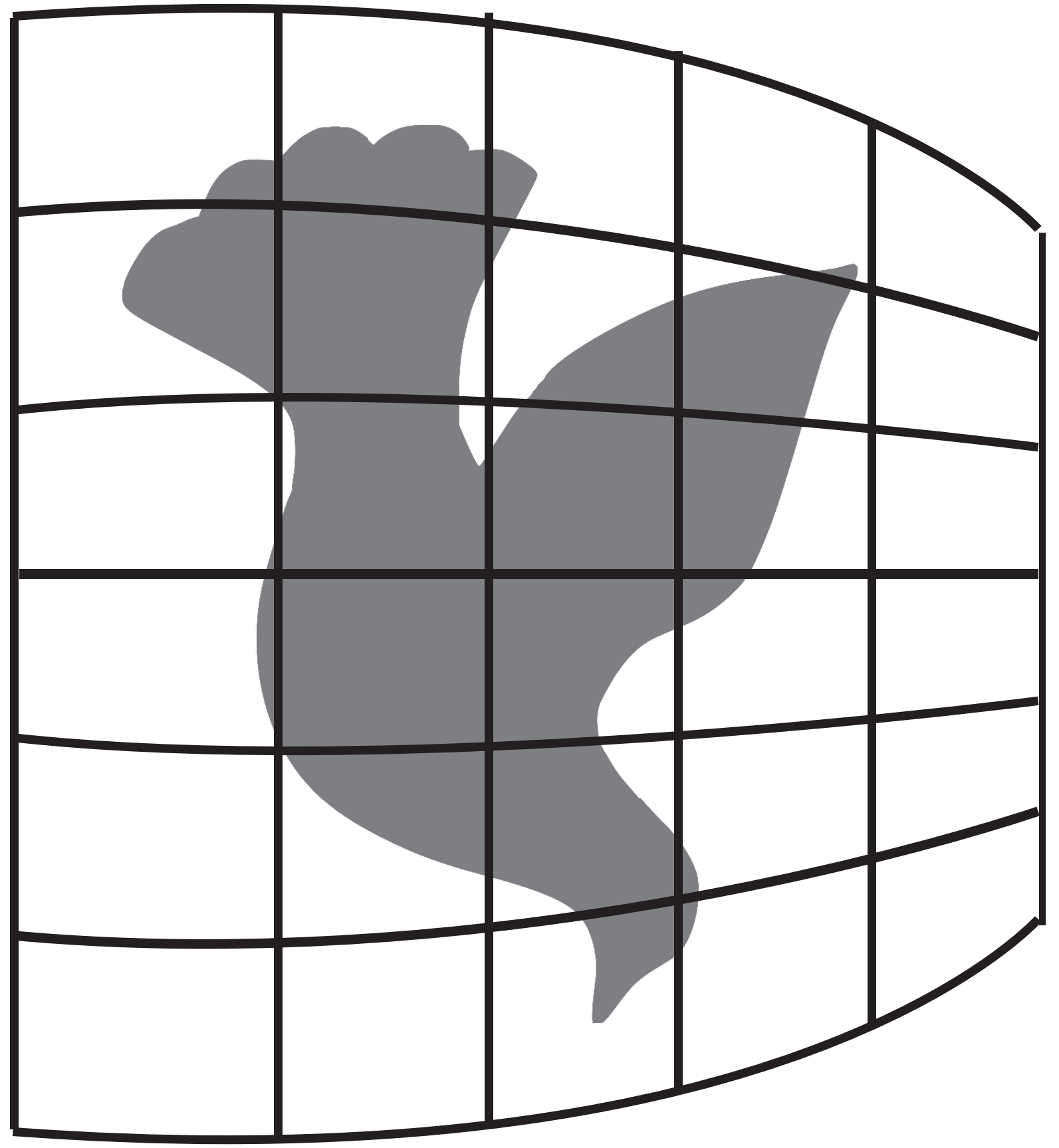

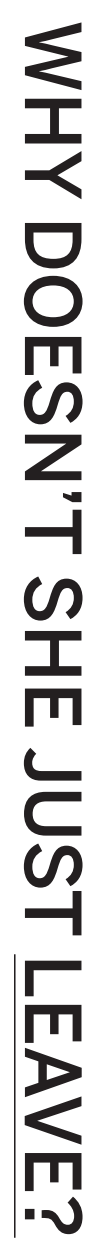


Appendix C:

PowerPoint Presentation Slides

(with notes pages) 


\section{PART TWO}

\section{Routine screening for IPV}

\section{Provider Training \& Planning Session \\ [insert name of institution] \\ [insert training date]}

Emphasize to participants that the day's session is a 'training and planning' session.

It is designed to be an interactive session geared toward mutual learning: The trainer,

on the one hand, will build participants' capacity to screen for IPV. The participants,

on the other hand, will lend their expertise toward planning for IPV screening in their

individual departments/settings (as they are likely to be better-versed in how their

work contexts operate than the trainer).

\section{Session One}

Intimate Partner Violence

What is it, anyway - and how can we help 


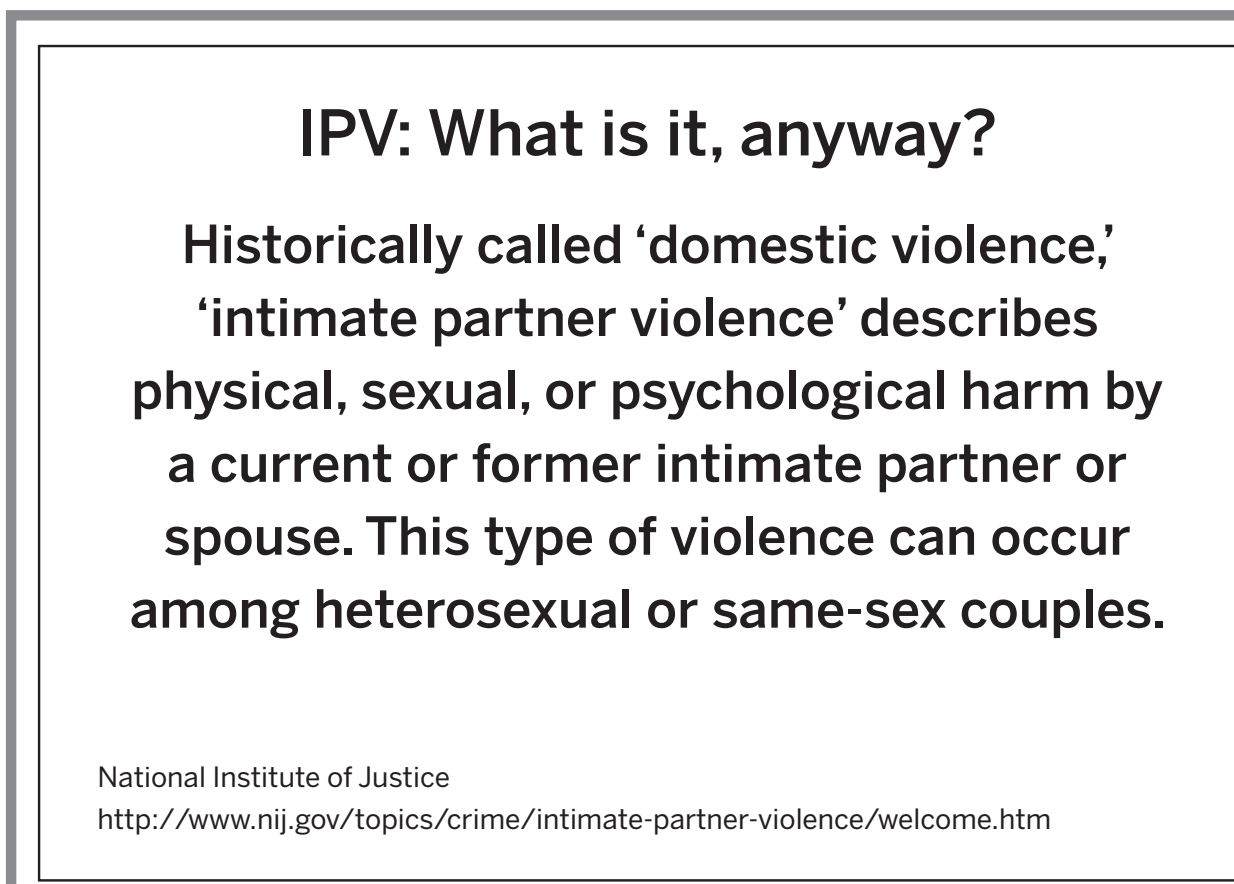

Notes:

Give participants a minute to reflect on the definition of IPV.

Ask participants what first comes to mind when they think of the term 'domestic.' Invariably, a participant will mention that they think of a house/home. Explain that for this reason, some experts argue that 'intimate partner violence' is a better term than 'domestic violence.' The latter sometimes leads to the assumption that this type of violence only happens among people who actually live together (typically, married or co-habiting couples), thus leaving out young people who may not be co-habiting, and ex-partners

who may still be abusive after a relationship is over.

Point out that IPV can also include economic violence/abuse, which is rarely talked about, but which is an important form of IPV from the perspective of survivors. Economic violence is a form of abuse that involves one intimate partner having "control over the other partner's access to economic resources, which diminishes the victim's capacity to support him/herself and forces him/her to depend on the perpetrator financially' https://en.wikipedia.org/wiki/Economic_abuse.

The definitions of all the kinds of IPV mentioned on the slide can be found at the link on the slide.

\section{Routine screening for IPV}

\section{One of "the most important} contributions [providers] can make to ending abuse and protecting the health of its victims is to identify and acknowledge the abuse."

(Council on Ethical and Judicial Affairs, A.M.A, 1992)

\section{Notes:}

Read the quote out loud.

Point out to participants that routinely screening for IPV (rather than not asking about abuse unless symptoms are evident) is one way of identifying this kind of violence, protecting the health of survivors, and making an important contribution as providers.

Highlight the fact that this statement was written in 1992 by the American Medical Association. This means that our settings in sub-Saharan Africa are comparatively far behind when it comes to the practice of routine screening, and to recognizing its utility.

Ask participants to estimate how many of the clients they see each week/month might be experiencing IPV, from what they can observe. Then have them reflect on how many more clients would be identified and connected to care if they were proactively asked about IPV at each visit. 


\section{IPV screening: Why bother?}

- IPV too common, too serious to remain unidentified

- Inquiring about obvious indications of violence no longer considered sufficient

- Can lead to early detection

- Provision of optimal care warrants that clients be asked routinely

- It's a preventive measure

- Etc.

Notes: This slide presents the rationale for implementing IPV screening protocols. Bullets 1-3: We now know that IPV is the most common form of violence experienced by women globally [the specific figures for the country concerned can also be inserted on the slide]. Given the serious health consequences associated with IPV, there is growing recognition in the region that providers must be proactive about identifying IPV survivors as soon as possible. It is no longer enough to only ask clients these kinds of questions when there are clear, physical signs that they have experienced IPV. The health consequences provoked by IPV make it critical for survivors to be identified or for IPV to be detected as early as possible. Routine IPV screening can help with this.

Bullet 4: IPV screening is also increasingly acknowledged as a quality of care issue. As IPV can often be the root cause of a client's health issue, not inquiring about it undermines the quality of care offered to the client.

Bullet 5: Routine screening for IPV can serve as a preventive measure against this form of violence. The very act of screening routinely conveys a message to clients that experiencing this form of violence is abnormal and has health consequences. Similar to routinely taking a client's blood pressure (which conveys a message about the danger of one's blood pressure becoming elevated), routine screening for IPV conveys a message about the danger of this experience, and about the need to seek care as a result.

Bullet 6: These are just some of the reasons why we need to bother about IPV screening. There are many more reasons which time may not permit us to delve into.

\section{What we want to achieve}

The routine screening of every female client who seeks services at the [name of screening site]

The successful referral to the GBV clinic of as many IPV survivors identified as possible 


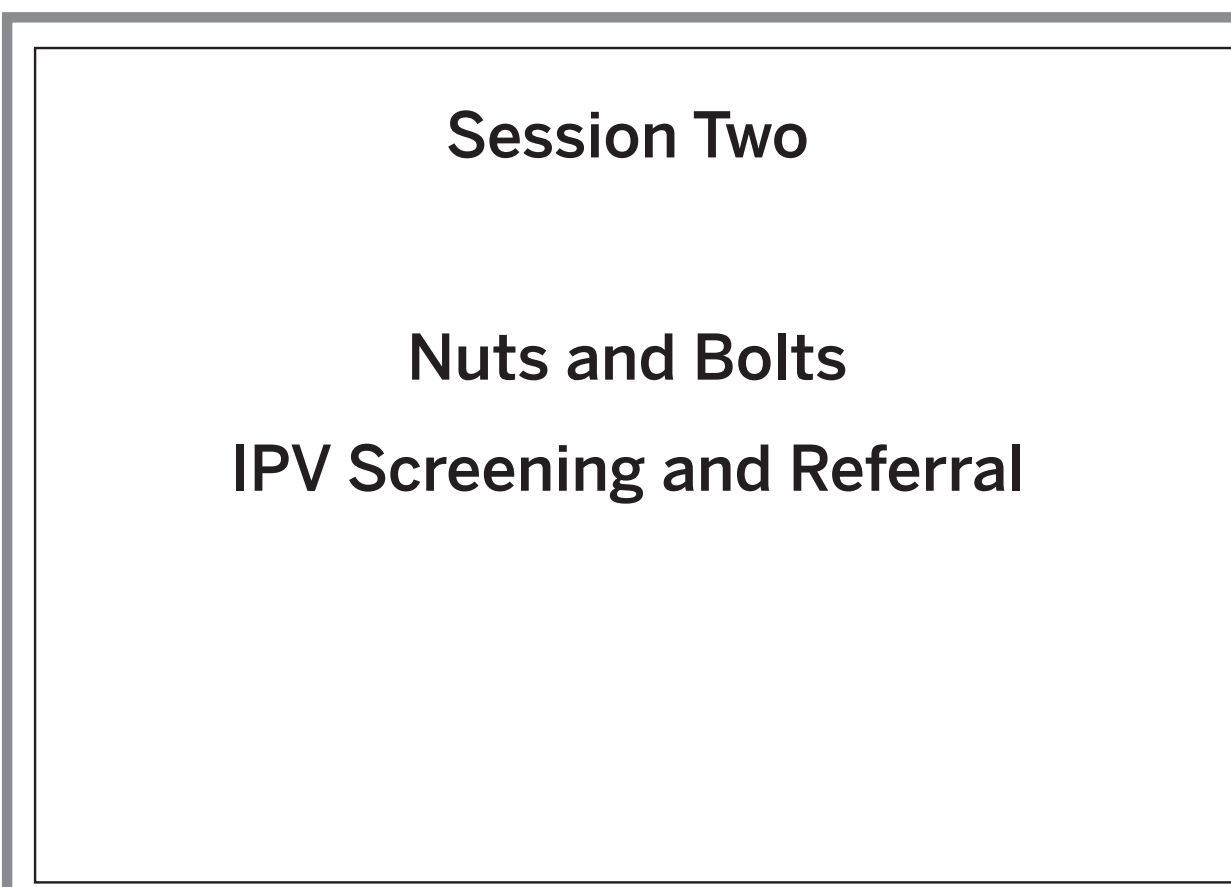

Notes:

\section{Screening}

\section{Remembering to screen}

- Posters in screening sites to remind providers to screen ("Have you asked?")

- Bright color on screening tool as a further reminder about routine inquiry

- Protocols or algorithms clearly describing appropriate identification and referral within each department

Notes: One major barrier to screening is actually remembering to screen.

Bullet 1: To support providers in the screening process, each provider carrying out IPV screening will have a simple A3 poster placed on the wall above their desk, with the following unobtrusive statement on it: 'Have you asked?'

Bullet 2: Draw participants' attention to the screening tool in their training folders.

Point out that the screening tool is framed in bright red as another means of reminding providers to screen clients routinely.

Bullet 3: To encourage routine screening, it is also important to have an algorithm that clearly helps providers remember the steps to be taken to prepare each screening site for the exercise, and to ensure privacy during actual screening.

Direct participants to the 'IPV screening: Figuring out critical/clinical pathways' handout in their folders. This document is left intentionally in draft form as each screening context will differ, and providers will have to decide how IPV screening will occur. Use this opportunity to review the hand-out with providers in the same department (if providers are from more than one department, put them in department-specific groups to encourage group thinking on what the clinical pathways should be).

Discuss with participants: 1 ) where clients can realistically be screened with complete privacy in their individual clinics/departments (this will serve as the "departmental room' mentioned in the draft IPV screening algorithm); and 2) which cadre of providers in their clinics/departments will realistically be able to conduct routine screening. 


\section{Remembering to screen: An important reminder}

"One woman, admitted to a trauma unit for serious injuries that resulted from battering, told [an IPV] advocate that it was only after her physician had asked her about abuse every day for six days that she felt safe enough to tell him what had happened. It took his repeated inquiry to convince her that he must really care and that she could trust him. She said that if he hadn't asked that last time, she probably would never have told him."

Warshaw, C. (1998). Establishing an appropriate response to domestic violence in your practice, institution and community, p. 121. http://www.ecu.edu/tnwe/Endowment/Resources_files/improvinghealthcare_4.pdf

Notes:

Routine screening for IPV might sometimes seem far too 'routine.' Providers may sometimes wonder if routine screening is 'worth it' if they do not identify as many clients as they would like through screening. However, success in routine screening should be defined (as least partly) by a provider's ability to screen routinely, regardless of the outcome.

Give participants a minute to absorb the quote on the slide. Emphasize that a survivor may not necessarily be ready to disclose violence on the first day she is asked about it. This is all the more reason why routine screening is important. If a provider gives up on routine screening and refrains from inquiring, this will amount to missed opportunities for the clients who are finally ready to disclose on that particular day.

\section{Screening}

- Screening appropriately: General tips for IPV screening

\section{-What if a survivor doesn't self-identify?}

"“ 'An abused woman is in a process. She will move through that process when she has sufficient strength and safety to take that next step. It is important to accept where she is in that process, even though you may not agree with her decisions and may fear for her safety' (Hadley, 1992). This has been the most difficult concept for both health care providers and advocates to

learn and incorporate."

\section{Notes:}

Bullet 1: Use the following questions to generate discussion among participants: What qualities or characteristics should a provider possess in order to carry out IPV screening effectively? What qualities or characteristics should the location of IPV screening have in order for this exercise to be carried out effectively? Draw participants' attention to the 'General Tips for IPV Screening' handout in their training folders. Walk through the handout with participants, highlighting the points that participants already pointed out independently during the discussion. Then, highlight other points which participants did not mention, perhaps asking how these point relate to their own work contexts.

Bullet 2: Give participants a chance to read the second bullet and comment on it.

Reiterate that 'success' with regard to routine screening for IPV can be viewed in severa ways. From a provider's perspective, success should be measured by (among other things) a provider's ability to properly and consistently screen clients, and refer them for care as needed. It will take time for some clients to open up to providers, hence the need for screening to be carried out routinely. Providers should not feel discouraged if, despite their best screening efforts, few clients are identified - particularly in the early days of any IPV screening exercise. 


\section{Introducing the screening tool}

\section{- Screening tool color(s)}

- Screening preamble

- Screening questions

- Defining 'IPV-positive'

- Referral section of the screening tool

Notes:

Bullet 1: Ask participants to pull out the IPV screening tool from their training folders. Point out that the bright color is deliberately chosen to help remind providers to screen, as mentioned previously.

Bullet 2: Have participants review the screening preamble. Ask participants why they think this preamble is particularly important. After some discussion, emphasize that the preamble: 1) helps ensure that clients do not feel individually 'targeted' by the new IPV screening protocol, but are helped to understand that all clients are now being asked these questions; 2) helps clients understand that violence is a health issue that needs to be identified and addressed as part of their overall health situation; 3 ) helps clients feel comfortable with the screening process, as it informs them that violence is common and that help for it is available; and 4) helps address any concerns about confidentiality, given the sensitivity of the questions.

Bullet 3: Give participants a chance to review the screening questions to make sure they are clear to the providers, and that providers feel that they would be clear if posed to clients. This process may result in the screening questions being collaboratively tweaked by providers to ensure that they are as intelligible as possible for clients.

Bullet 4: For the purposes of the IPV screening exercise, a client is to be considered as IPV-positive if she answers 'yes' to any of the screening questions posed.

Bullet 5: The client's answers to each question should be ticked (whether 'yes' or 'no'). If a client answers 'yes' to any one of the questions, they should not be asked the questions again. They should be given a referral that day, and the date of this referral should be noted in the space provided.

\section{Referral}

- GBV clinic contact info in all screening sites

- The use of client accompaniment services

- Ensuring clients' rights are respected, while keeping the referral process convenient for those who are amenable

- The process of receiving referrals at the GBV clinic

- IPV clients to be given first priority?

- Emergency counseling coupled with a later date appointment?

Notes:

After screening and identifying an IPV-positive client, refer the client for gender-based violence (GBV) services. In most health facility contexts, referrals are made by using a

referral slip that indicates where the client was referred from, on which date, where the client

is being referred to, and why. This process can be used to facilitate IPV referrals as well. In addition, there are other components that can be incorporated to help enhance the referral process, especially given the sensitivity of IPV. Have participants talk through what would work for their individual contexts, based on the points below:

Bullet 1: All sites where IPV screening is occurring, and all providers conducting such screening should have the phone number of the GBV clinic where clients are being referred readily available. In some contexts, departmental phone numbers may be harder to get through to, or may simply be out of order. If so, mobile numbers can be used instead, if this is acceptable. Having such contact numbers helps ease the referral process: for example, the initiating department can place a call to the receiving department to alert them that a survivor has been referred and is on the way. Or, survivors who may not have the time to head to the GBV clinic right away can make a phone call later to set up an appointment.

Bullet 2: Clients should be accompanied by someone from the initiating department to the receiving department to ensure clients are not lost during the referral process. Health facilities often have resources that could be tapped into for this purpose. Students in training, interns, auxiliary workers, etc., can step in to play this role. Once identified, this cadre of health facility affiliates should be sensitized on GBV issues before playing a survivor accompaniment role. It is also important to emphasize that some clients may not want to be escorted, and that they are within their rights to decline this service.

Bullet 3: A discussion about what will realistically happen to clients once they get to the GBV clinic is also important. Will these clients be given first priority? Is it more realistic to expect that they will receive some sort of emergency counseling and are then given a later appointment (i.e.., if there are no health implications) for more comprehensive care? Slide 13 will help delve further into what the GBV clinic concerned can realistically provide. 


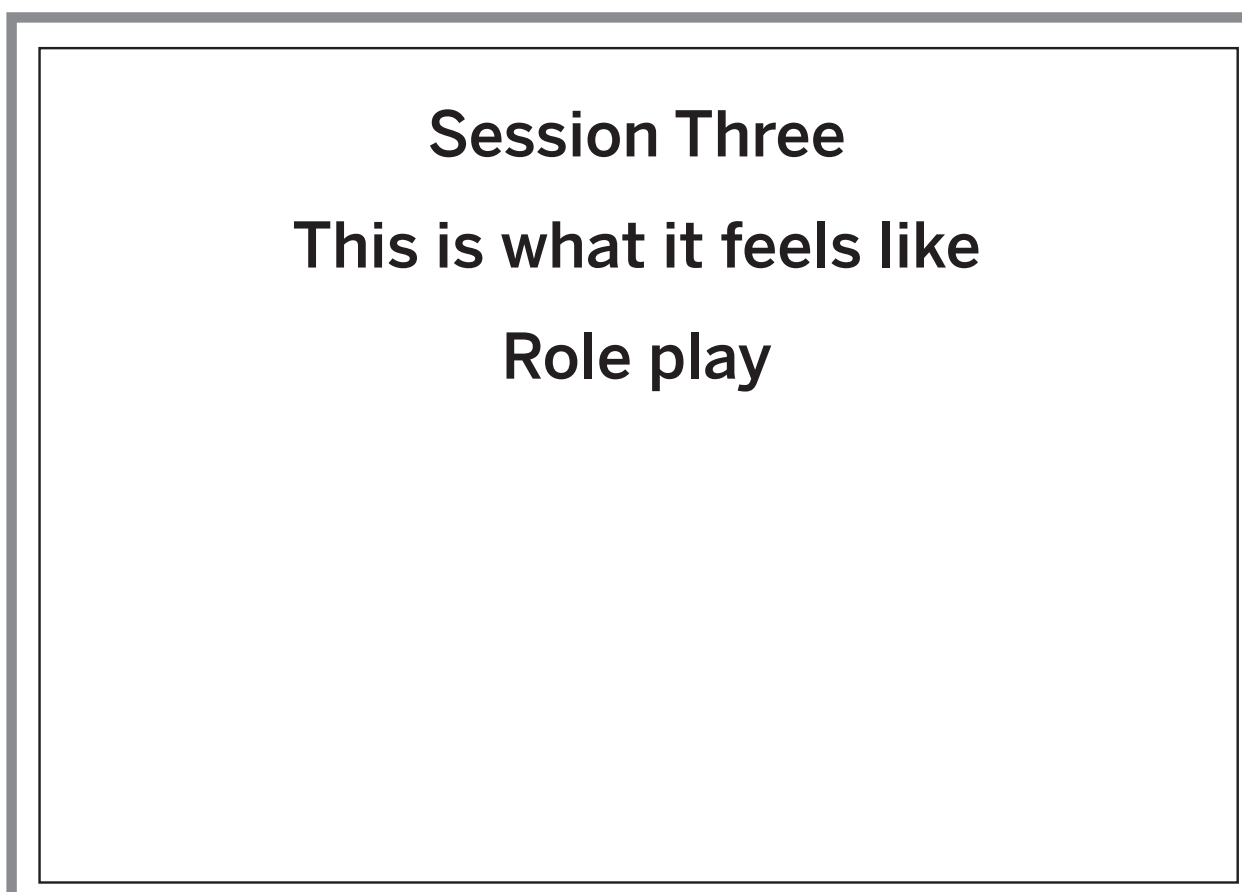

Notes:

\section{Screening Role Play \& Discussion}

- In groups of 3: The roles of a provider, client, and observer are to be issued to group members. All 3 group members (especially the observer) are to take note of anything remarkable about the screening process.

- How did you feel ....

- as a provider, asking these questions?

- as a client, being asked these questions?

- What did you notice, as an observer, about the process?

- Other observations?

\section{Notes:}

Bullet 1: Ask participants to get into groups of 3. Each group should select a "provider,' 'client,' and 'observer' for the role play exercise. The provider asks the client the screening questions. The client responds to the screening questions. The observer notes down their impressions about how the process went. The provider and client should also make mental notes of what it feels like to be in the position of a provider or client during an IPV screening exercise. Group members should take turns until every member of the group has had a chance to play the role of either a provider, client, or observer

Bullet 2: Bring the groups back into plenary, and have participants reflect on how they felt being in the positions of provider and client. Participants should also share their observations about the screening process. 


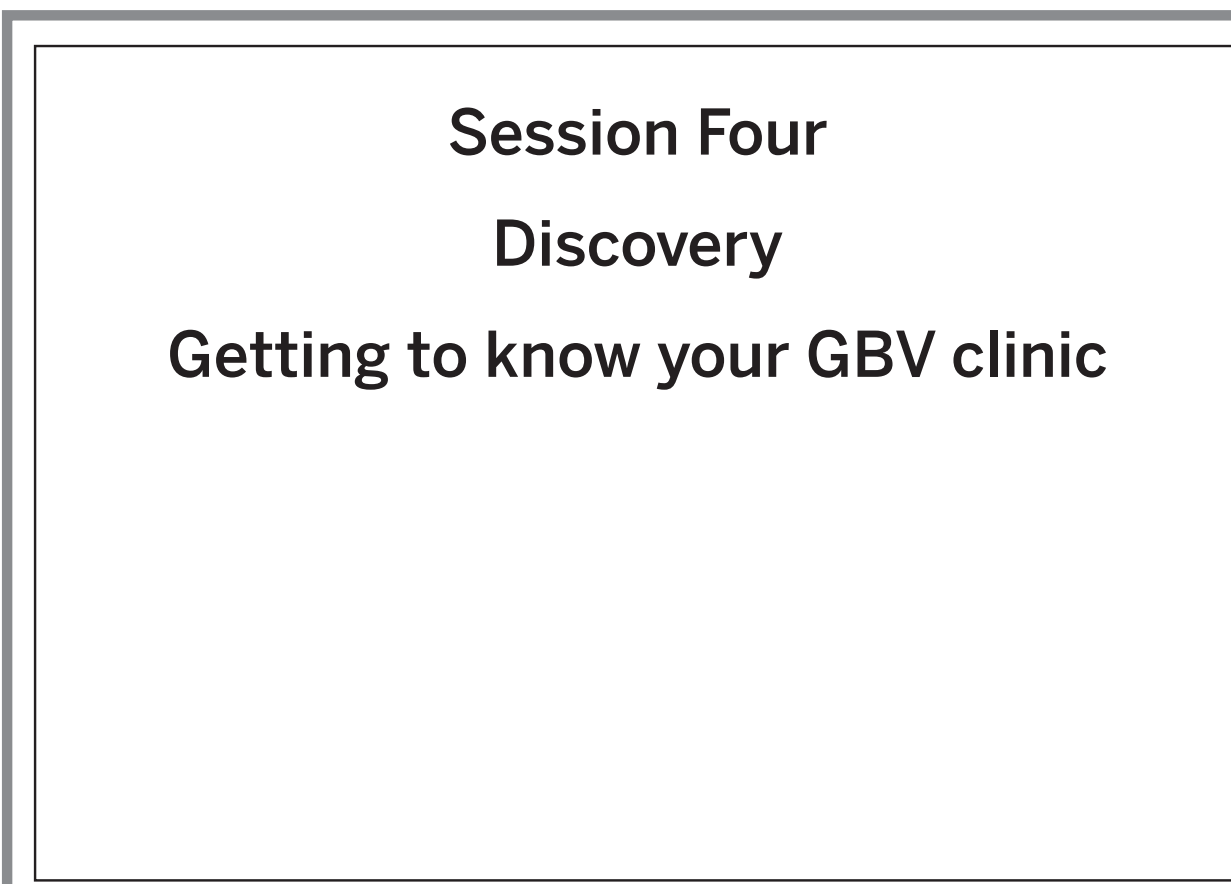

Notes:

\section{Session Five}

Planning to act

Developing an action plan

for routine IPV screening

Notes:

Ask participants to group themselves according to their clinics/work sites (if applicable).

Draw participants' attention to the Action Plan for IPV Screening Worksheet in their training manuals. Walk them through the worksheet, explaining that each clinic/work site now needs to prepare for the actual introduction of routine IPV screening in their setting. The worksheet is meant to help them think through the resources (human

and otherwise) that their work contexts have for supporting routine IPV screening and the potential barriers to this process in their individual work settings. Group members should hold discussions on each section of the worksheet and work collaboratively to fill it out.

Bring participants back into plenary and have a representative for each group summarize the group discussions. Feedback from this first group may raise interesting and pertinent issues and ideas related to the realities of screening which subsequent groups can also speak to. Potential barriers to screening can be resolved by the wider group.

Remember to gather all the worksheets at the end of the exercise. They can be referred to once routine IPV screening begins in order to retrieve names of influential staff

members, staff members who should be part of the next training session (if any), and solutions to barriers as they come up during screening program implementation. 


\begin{tabular}{l} 
Session Six \\
What we've learned and how it went \\
Closing \\
\hline \\
Wotes: \\
As participants to retrieve the evaluation form from their training folders and to kindly \\
fill tout. Gather all the evaluation forms at the end and use participants' comments to \\
inform future training sessions.
\end{tabular}

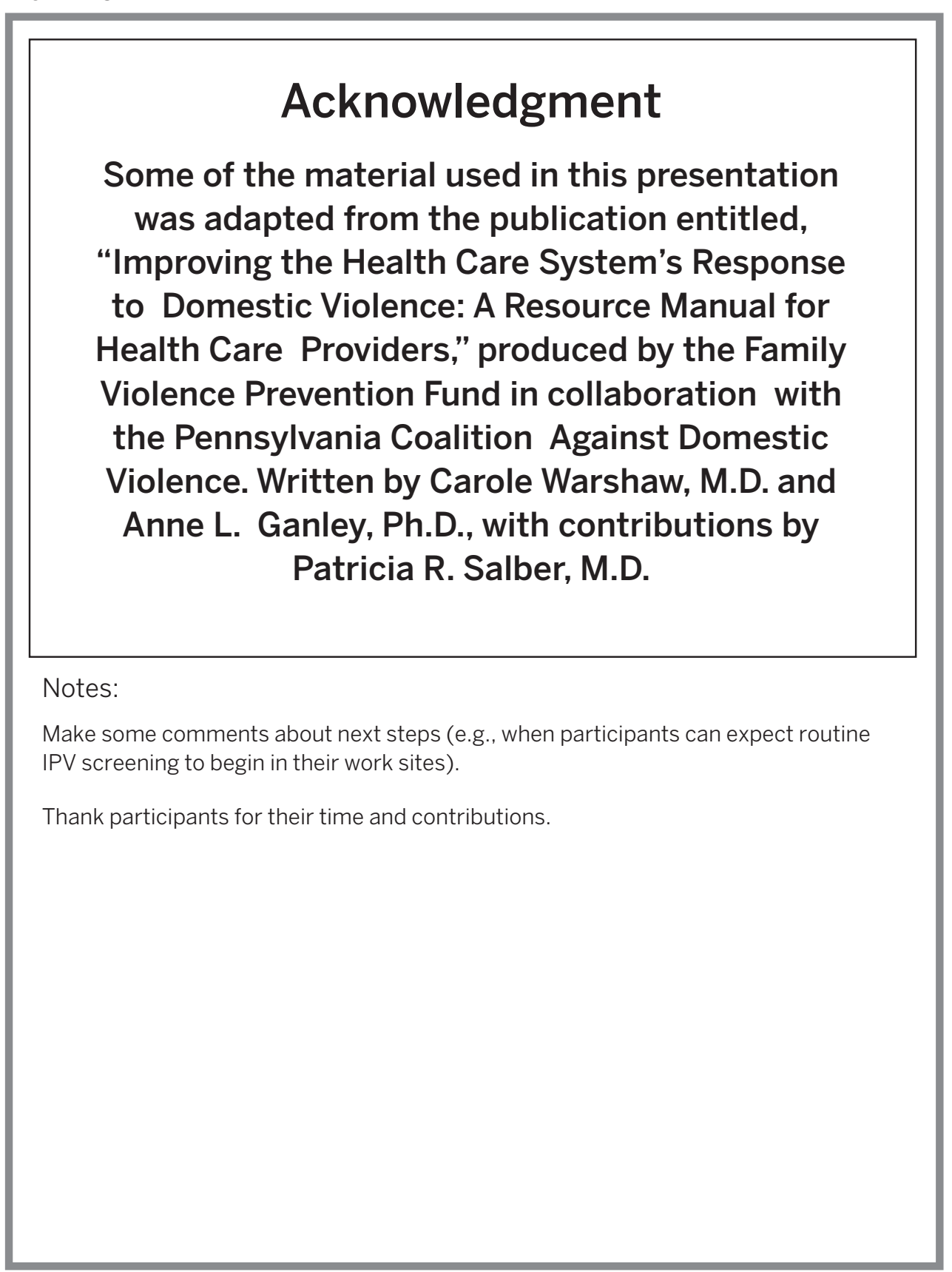




\section{Appendix D: Hand-outs}

Hand-out \#1: Training and Planning Agenda

Hand-out \#2: IPV Screening: Figuring out Critical/Clinical Pathways

Hand-out \#3: General tips for IPV screening

Hand-out \#4: IPV screening tool

Hand-out \#5: Action plan worksheet

Hand-out \#6: Evaluation form 

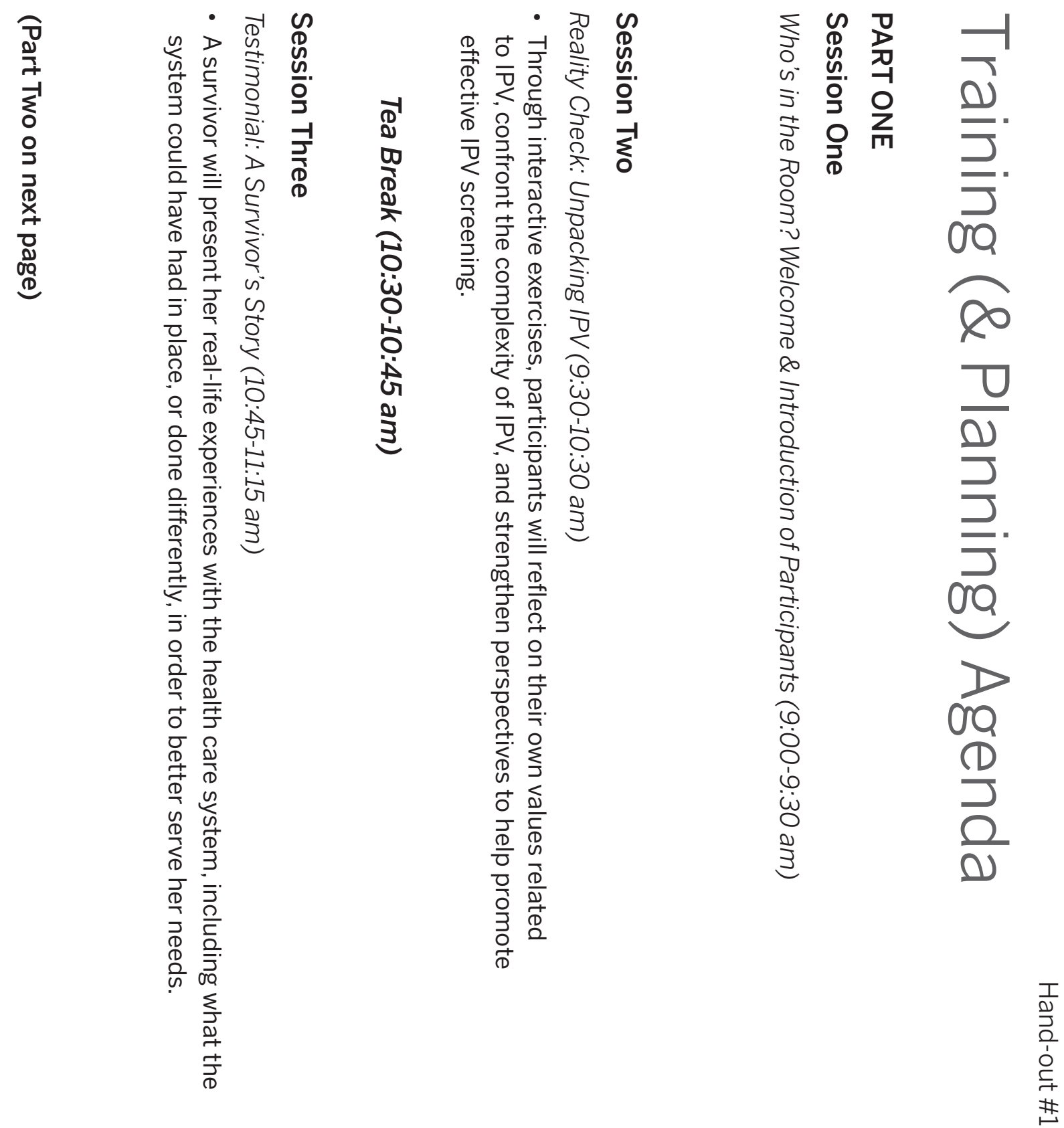

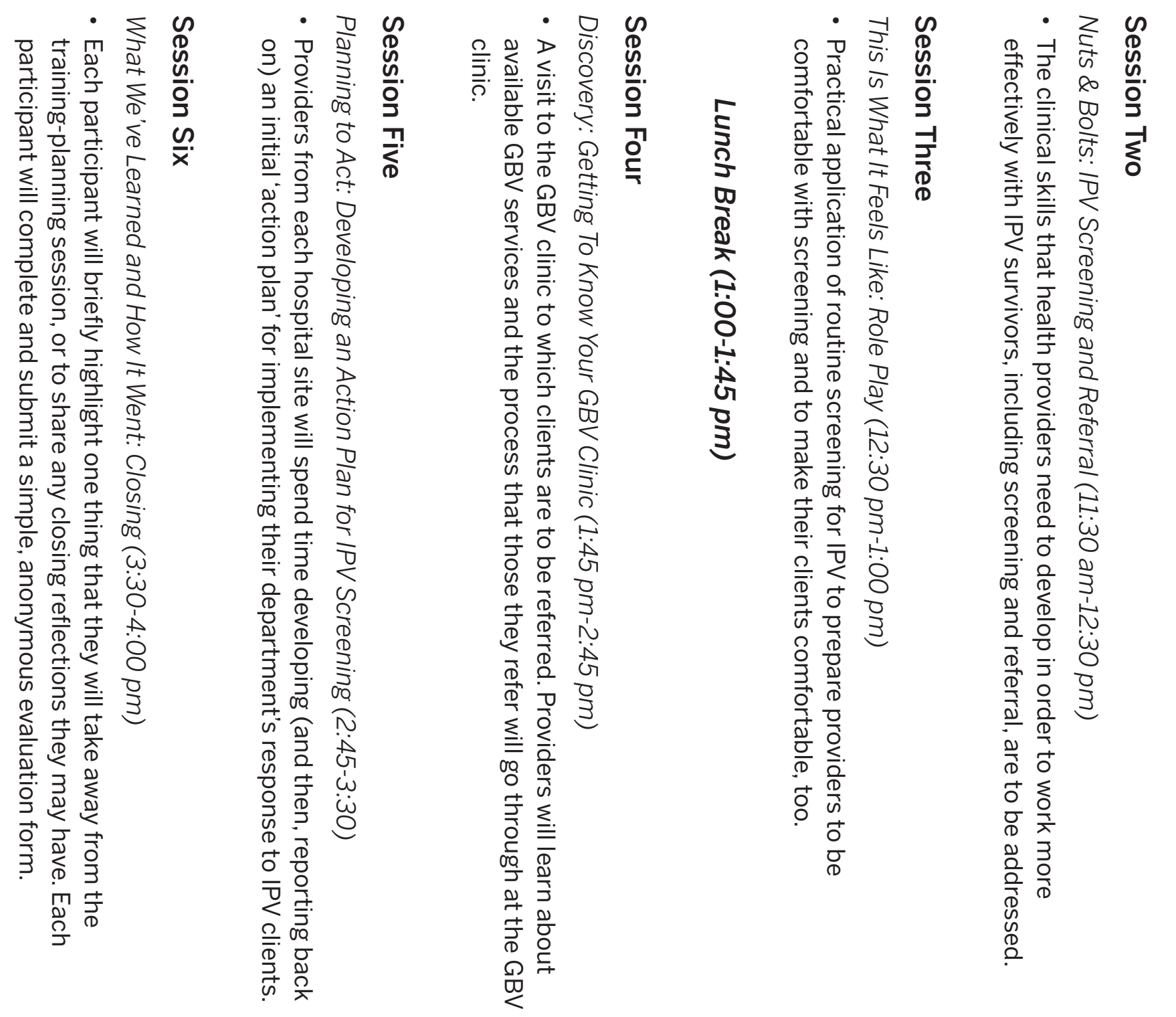

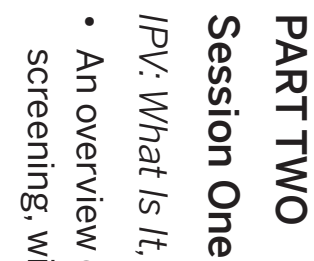
齐亦 চ 응 है 유ㅇㅡㅡ 을 임

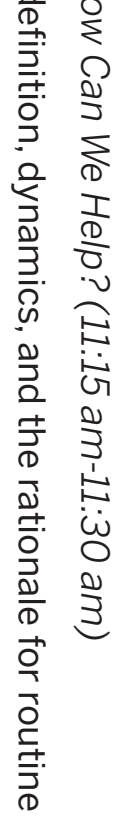




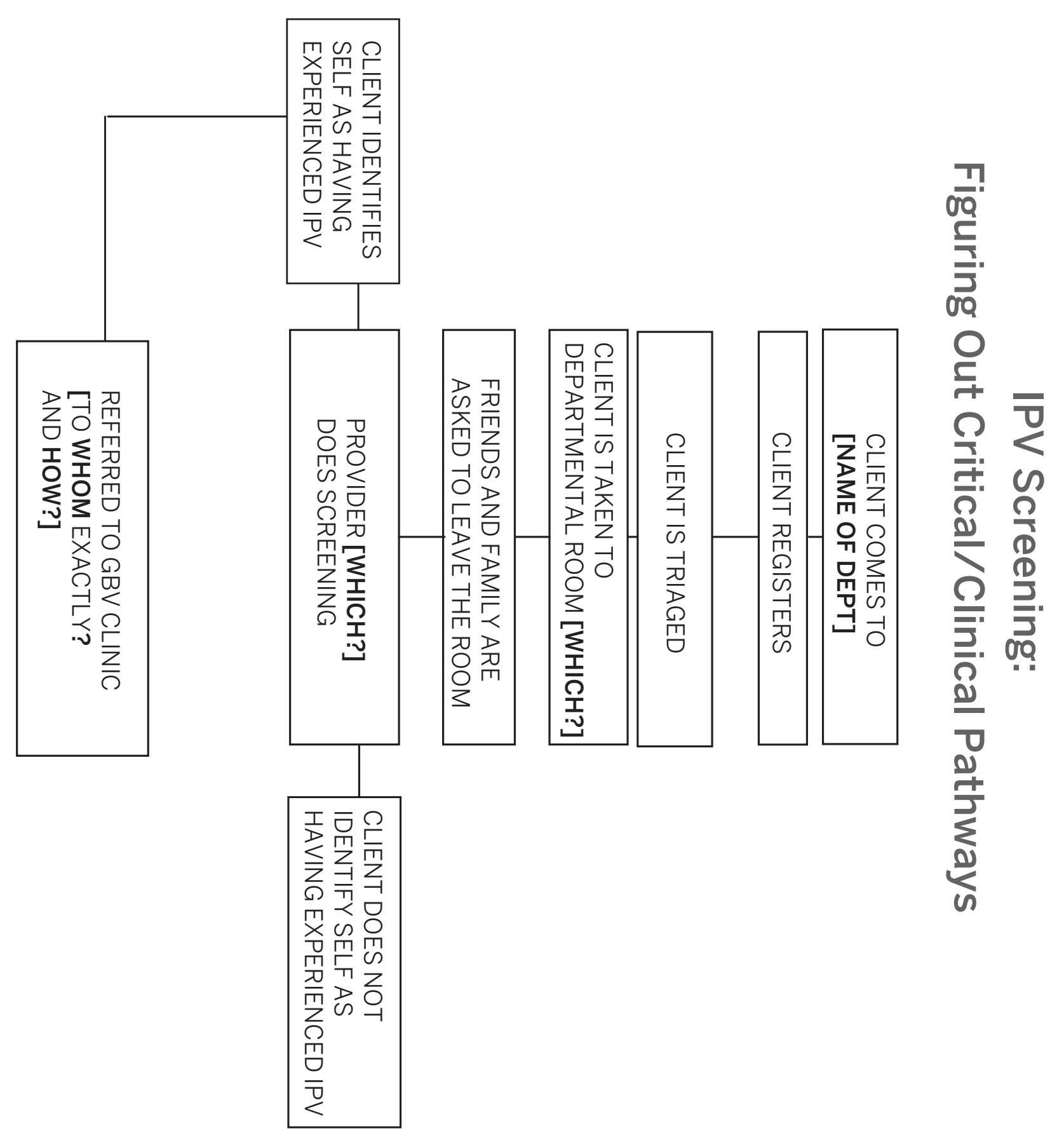



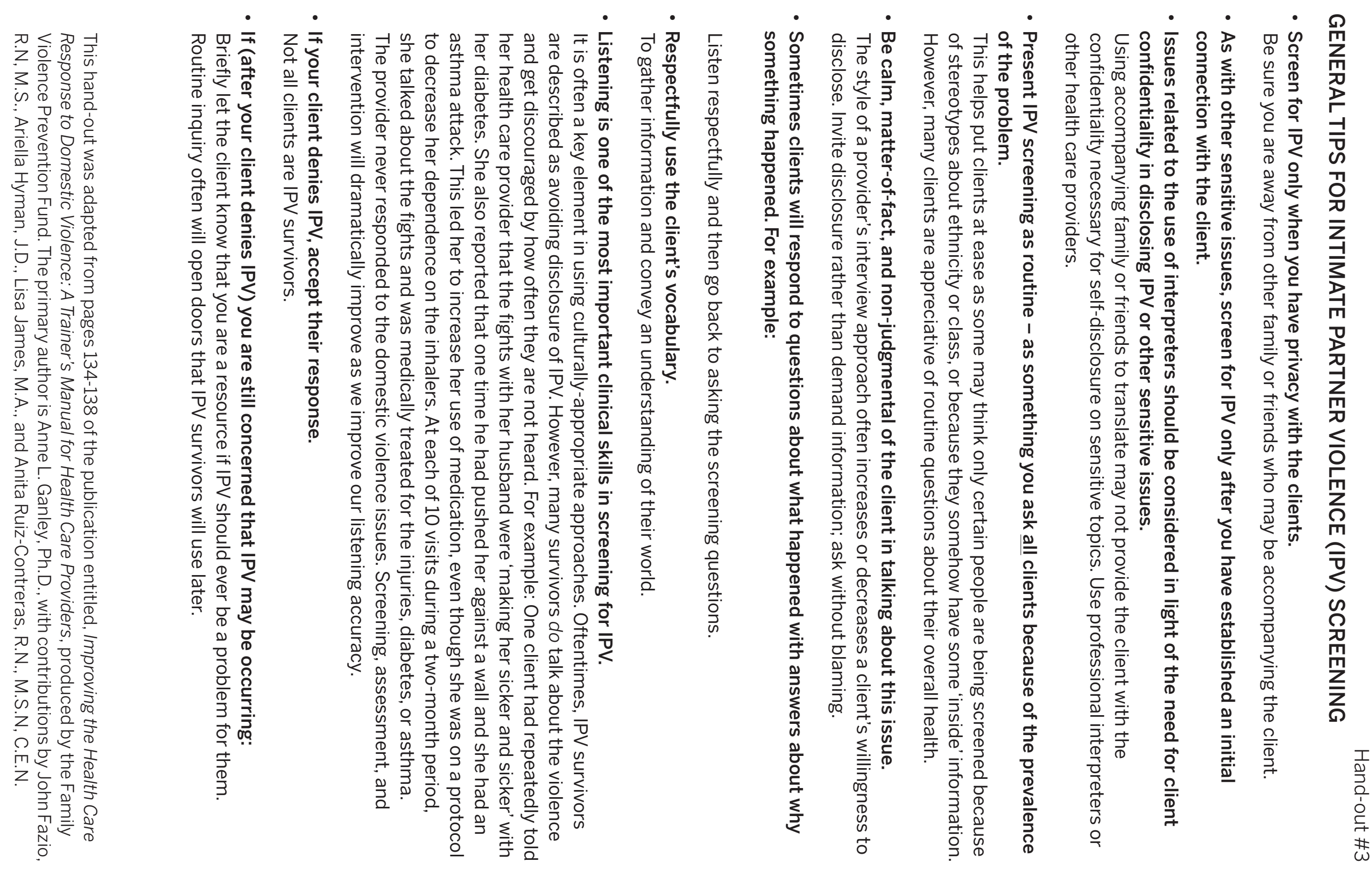

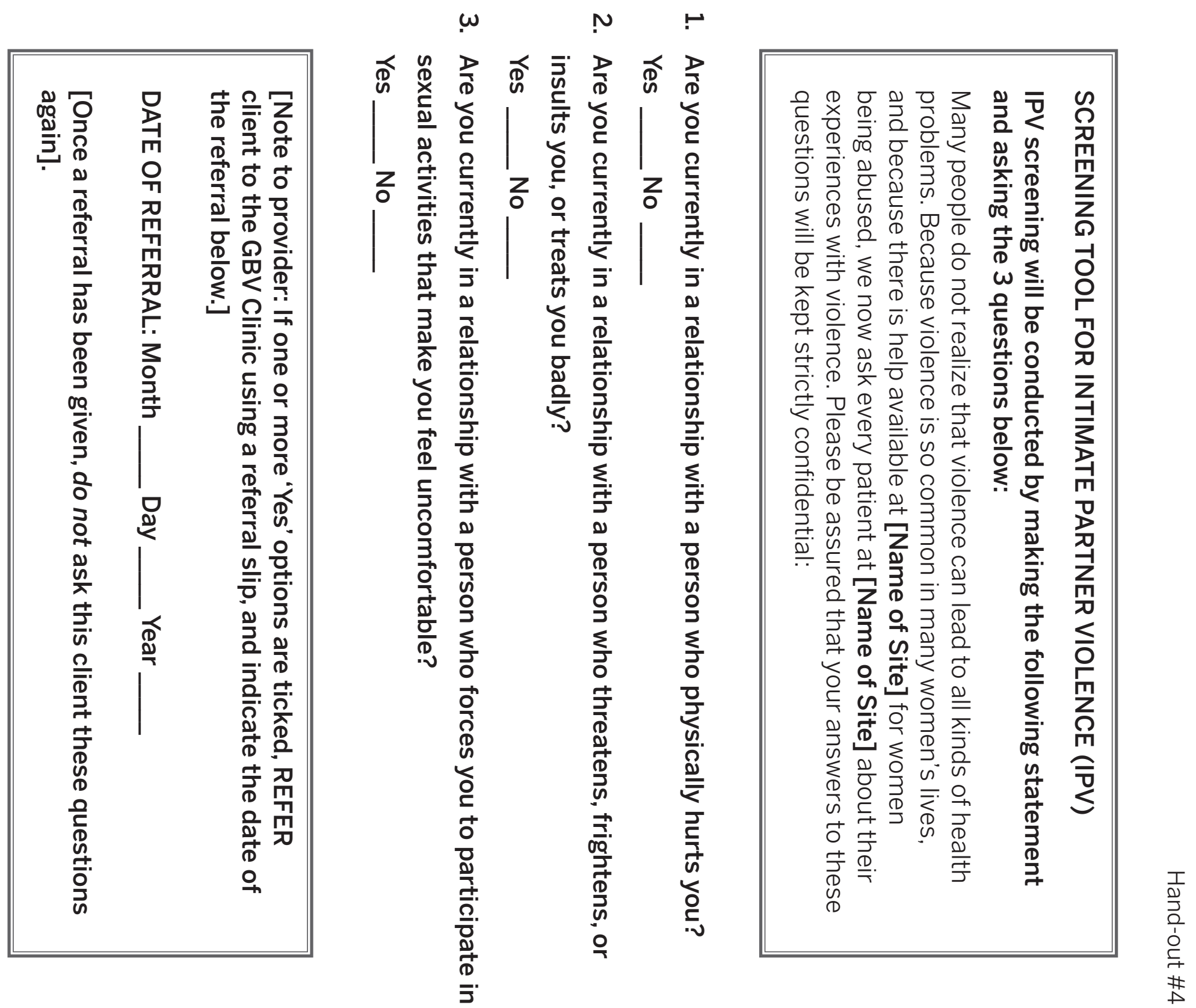


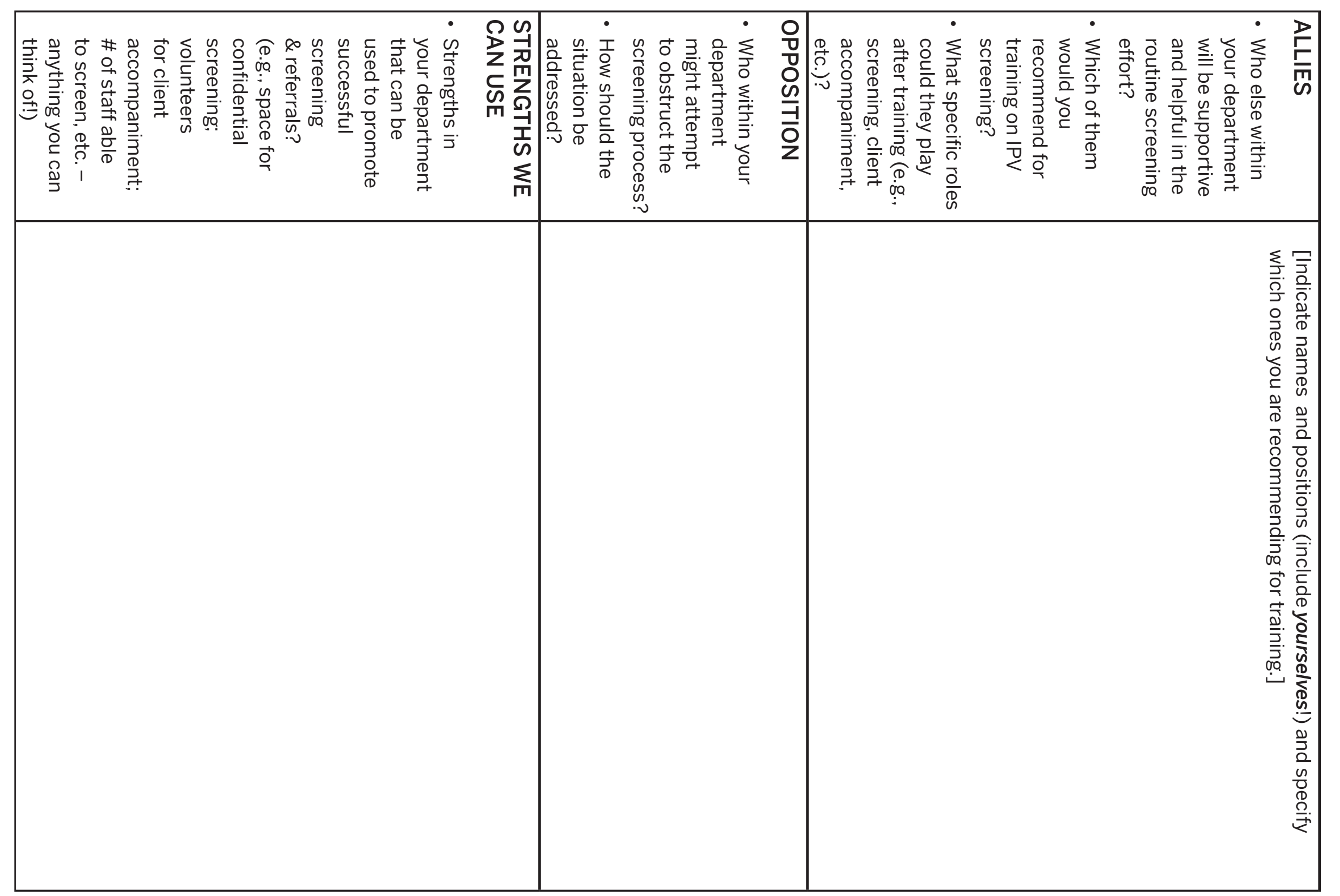

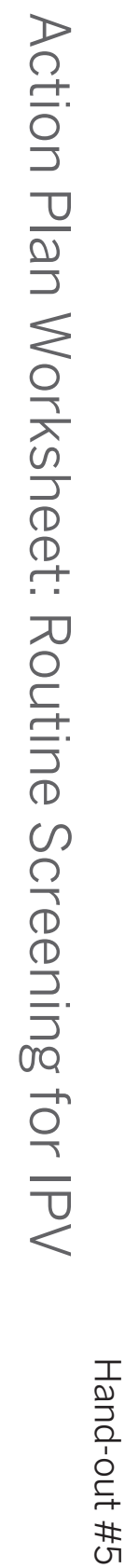



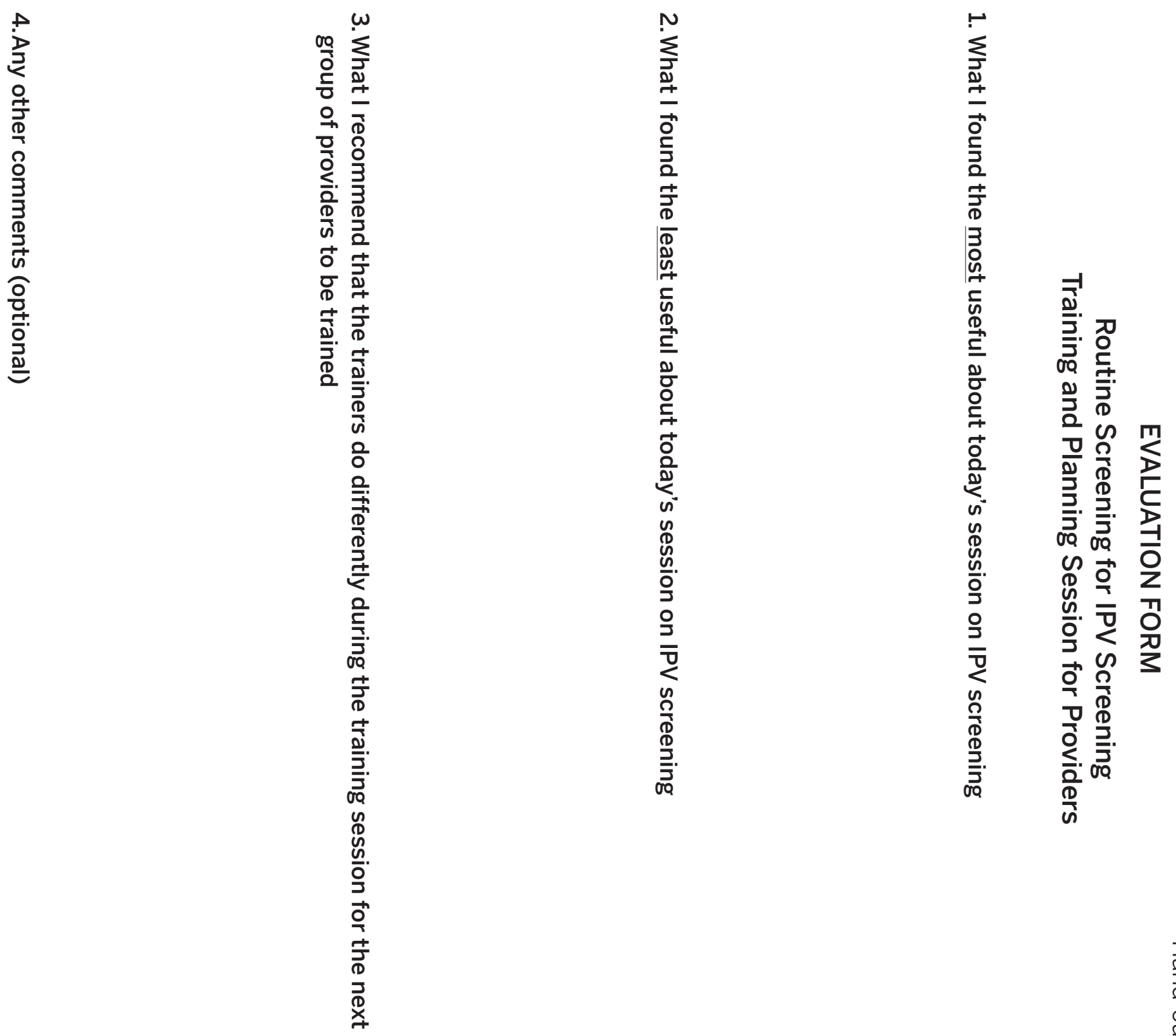

East, Central and Southern

Africa Health Community

Fostering Regional Cooperation for Better Health 